\title{
Euclidean Actions, Instantons, Solitons and Supersymmetry
}

\author{
T. Mohaupt册 and K. Waite \\ Theoretical Physics Division \\ Department of Mathematical Sciences \\ University of Liverpool \\ Liverpool L69 7ZL, UK
}

\begin{abstract}
Theories with axionic scalars admit three different Euclidean formulations, obtained by Wick rotation, Wick rotation combined with analytic continuation of the axionic scalars, and Wick rotation combined with Hodge dualization. We investigate the relation between these formulations for a class of theories which contains the sigma models of $N=2$ vector multiplets as a special case. It is shown that semi-classical amplitudes can be expressed equivalently using the two types of axionic actions, while the Hodge dualized version gives a different value for the instanton action unless the integration constants associated with the axion fields are chosen in a particular way. With this choice the instanton action is equal to the mass of the soliton or black hole obtained by dimensional lifting with respect to time. For supersymmetric models we use the Euclidean supersymmetry algebra to derive a Euclidean BPS condition, and we identify a geometrical criterion which distinguishes BPS from non-BPS extremal solutions.
\end{abstract}

\footnotetext{
*Thomas.Mohaupt@liv.ac.uk
}

$\dagger$ K.Waite@liverpool.ac.uk 


\section{Introduction}

Instantons and solitons provide important analytical tools to study non-perturbative effects of field and string theories. Often they can be related to one another via dimensional reduction over time. One application of this link is to generate stationary solutions, for example black holes, by lifting solutions of the time-reduced theory [1, 2. In the context of supergravity and string theory instantons, or $(-1)$-branes, can act as seed solutions to generate all the solitons, or $p$-branes with $p \geq 0$. Euclidean actions constructed via reduction over time are in general not positive definite, which raises the question whether their solutions, while definitely useful for constructing solitons via lifting, can really be interpreted as instantons. A related problem is that upon substituting the 'candidate instanton' into the Euclidean action one obtains zero instead of a finite positive value needed for a consistent semi-classical approximation. These problems are not tied to dimensional reduction over time, but always occur when looking for instanton solutions supported by scalars in more than one dimension. The reason is that Derrick's theorem forbids purely scalar 'instantons' (nonconstant finite action solution) and 'solitons' (non-constant time-independent finite energy solutions) in two or more space-like dimensions [3, 4, 1$]$

It is well known that there is a class of axionic instanton solutions which circumvents this no go theorem, in particular axionic wormhole-type solutions [5, 6, 7, 8, the D-instanton solution of type-IIB supergravity [9, 10, 2 and hypermultiplet and vector multiplet instanton solutions in $N=2$ string compactifications [11, 12, 13, 14, 15, 16, 17. There are three different approaches to such axionic instantons. The first makes use of the fact that like finite dimensional integrals, functional integrals can be dominated by a complex rather than real saddle point [6, 7]. One then has to show that there exists a complex saddle point which leads to a consistent semi-classical approximation to the functional integral one wishes to compute. The tunneling through a circular potential barrier provides a simple quantum mechanical toy example for this type of solution [6]. Another approach is based on the fact that Wick rotation and Hodge dualization do not commute and uses a dual Euclidean action where the axions have been replaced by antisymmetric tensor fields [5, 6, 8, 9, 10. This Euclidean action is positive definite and has real saddle points, which is not in contradiction to Derrick's theorem because the tensor fields have a local gauge symmetry. At the level of individual amplitudes the tensor formulation can be related to the axionic formulation by a change of variables in the functional integral. However, the Hilbert spaces of axionic and tensor field theory are not isomorphic in general, 8 and the breaking of continuous axionic shift symmetries is not straightforward to describe. The third approach is to use an indefinite Euclidean action with inverted kinetic terms for the axions [9, 10]. For such actions Derrick's theorem does not apply and real saddle points exist. Actions with inverted kinetic terms for axions result from dimensional reduction over time, but can also be constructed by either applying Hodge dualization before and after the Wick rotation, or by using a modified Wick rotation, where axionic fields are continued analytically to imaginary values [18, 9, 19, 10. One

\footnotetext{
${ }^{1}$ Exceptions arise for models without a scalar potential if the Laplacian has non-trivial zero modes. The world sheet instantons of string theory are an example of this.

${ }^{2}$ The D-instanton solution corresponds to flat Euclidean space in the Einstein frame, but is a finite neck wormhole in the string frame.
} 
reason for using a modified Wick rotation for axions is Euclidean supersymmetry. Poincaré and Euclidean supersymmetry algebras have different R-symmetry groups, which in turn implies different geometries of their scalar target spaces. For four-dimensional $N=2$ vector multiplets it was already observed in [18] that the change

$$
U(1)_{R} \times S U(2)_{R} \rightarrow S O(1,1)_{R} \times S U(2)_{R}
$$

in the abelian factor of the R-symmetry group implies an analytical continuation of all vector multiplet axions. It was shown in [20, 17] that this can be understood geometrically as replacing the complex structure of the scalar target space by a para-complex structure. Based on this observation, Euclidean variants of the special geometries of both rigid and local vector multiplets have been formulated.

We remark in passing that it is perfectly consistent to use a Euclidean version of a supersymmetric theory which is not invariant under the Euclidean supersymmetry, as long as one can derive Euclidean Ward identities which give the supersymmetric Ward identities upon continuation to Minkowski space 21. Options for the Euclidean formulation of supersymmetric theories have also been discussed 19, 22, 23, 24, 25. In this paper we will not attempt to give a comprehensive discussion of the various existing approaches to Euclidean supersymmetry. Instead we focus on the specific properties of axionic instanton solutions for the Euclidean $N=2$ vector multiplets constructed in [20, 17, in particular the Euclidean BPS condition and its geometric interpretation.

The other main purpose of this paper is to clarify the status of indefinite Euclidean actions for axions, whether supersymmetric or not. We use the class of Euclidean sigma models introduced in [26, which includes the scalar sector of Euclidean $N=2$ vector multiplets as a special case. While it was demonstrated in [26] and 27] that these models have a rich set of solutions which can be lifted to extremal multi-centered and non-extremal single centered black hole solutions in five dimensions, we are now focussing on the question whether these solutions can be interpreted as instantons. As we will see this class of models is suitable for exploring the relations between the three types of Euclidean actions introduced above, because instanton solutions can be constructed in a systematic way. Two constraints are imposed on the scalar metric: (i) the metric must be determined by a potential, a feature which generalizes the geometry of $N=2$ vector multiplets, (ii) the continuous axionic shift symmetries must form an abelian group. This allows a simple lifting of the Euclidean theory with respect to time, and implies a simple and transparent structure of the solutions, which helps us to investigate the conceptual points we are interested in. These constraints admit models with an arbitrary number of scalar fields which can be treated in a universal way, and do not require the scalar target space to be a symmetric or homogeneous space. This is a reasonable compromise between generality and analytical control over solutions.

While the role of complex saddle points when using definite axionic actions, the use of the Hodge dual tensor field action as an alternative description of instantons, and the use of indefinite axionic actions in order to generate solutions by dimensional lifting have been discussed in detail in the literature, it has remained unclear which role indefinite axionic actions play in the context of instantons (as remarked in [28]). For our class of models we find a simple answer by re-investigating the amplitude calculation of $[7,28$ : amplitudes can be 
expressed both in terms of the definite and the indefinite action, and both formulations are related by a change of variables which is nothing but the analytical continuation of the axions which relates the two types of actions. While in one formulation the functional integral has an imaginary saddle point and one needs to integrate over real fluctuations around it, in the other formulation the saddle point is real and the fluctuations are imaginary. Given that the two actions are related by analytical continuation this might sound obvious, but, as we will see, the calculation of amplitudes involves the careful consideration of boundary conditions and boundary terms. In particular, since the naive bulk action vanishes when evaluated on instantons, boundary terms are essential for obtaining a consistent semi-classical approximation where instanton contributions are exponentially suppressed. As we will see, the boundary terms obtained are related by the same analytical continuation as the bulk actions, which is needed for both formulations being equivalent. Moreover, the amplitude calculation is instructive because it shows that for axionic instantons we cannot avoid to consider complex values of the axions. The situation is analogous to computing an integral over the real line which has a complex saddle point by contour deformation. Adopting a 'complex point of view' shifts the emphasis from the quest for 'the' correct Euclidean action to the more appropriate one of choosing the appropriate 'integration contour' for the semi-classical approximation of a given functional integral. A similar point of view is taken in [29] in the context of matrix models.

Since the dual tensor action is positive definite and has real saddle points, one might think that one could abolish the axionic actions altogether and only use the tensor formulation. However, there are problems with this idea. In the supersymmetric context there are cases, notably the dilaton multiplet in heterotic compactifications, where no appropriate dual off shell supermultiplet exists 31. More generally, it is not clear how to capture the breaking of continuous axionic shift symmetries in the tensor formulation. The reason is that while axions have global shift symmetries, tensor fields have local gauge symmetries, and therefore it is not clear how the breaking of continuous shift symmetries, which is believed to be a genuine physical effect, could be captured by a local effective scalar-tensor action. The program of finding the full non-perturbative corrections to hypermultiplet moduli spaces (see 32 for a review) faces precisely this problem. Therefore it is desirable to develop methods for computing instanton effects which do not rely on the tensor formulation.

This asymmetry between two theories which are completely equivalent classically, or, more precisely, at the level of their equations of motion, indicates that they are not fully equivalent as quantum field theories. This observation has already been made in a different context in 8 , where the relation between the Hilbert spaces of axionic and tensor field theories was investigated. By taking space to be compact and applying Hodge decomposition to the space of classical solutions, the precise relation between the Hilbert spaces of the two theories was worked out. It turned out that the Hilbert spaces are not isomorphic, but differ in their zero mode parts. The zero modes of the axions, while completely trivial in the classical theory, label superselection sectors of the quantum theory. This has no analogue in the tensor theory, which has a local gauge symmetry, and therefore the Hilbert space of the axionic theory is larger 3 We encounter

${ }^{3}$ However one can embed the axionic Hilbert space into multiple copies of the tensor field 
a different aspect of this non-equivalence (or 'equivalence up to zero modes') when re-investigating the relation between the semi-classical evaluations of the respective partition functions. Here we observe that in the axionic formulation the instanton action explicitly depends on the boundary values of the axions, which is a manifestation of the breaking of continuous shift symmetries. In contrast, the instanton action of the dual tensor field theory does not depend explicitly on two-form potential, as required by local gauge symmetry. As a consequence the instanton actions of both pictures only agree if the boundary values of the axions, which are integration constants of the instanton solution, are adjusted in a particular way. With this adjustment the instanton action is equal to the ADM mass of the black hole obtained by lifting.

In the last part of the paper we specialize to supersymmetric models. We show that the Euclidean BPS condition can be derived from the Euclidean supersymmetry algebra and that it is related by dimensional lifting to the BPS condition for a massive charged point-like BPS state. We also find a geometrical characterization of the distinction between BPS and extremal non BPS solutions in terms of the para-complex geometry of the target space, which generalizes to supersymmetric theories.

Throughout the paper we restrict ourselves to what we call extremal Euclidean solutions. The working definition of 'extremal' is a solution with vanishing energy momentum tensor. Such solutions remain completely unmodified when coupling the theory to gravity. This observation can be used to construct extremal black hole solutions in terms of a scalar sigma model on flat Euclidean space. Moreover, in the dual tensor formulation such solutions saturate a Bogomol'nyi bound, and in the supersymmetric case BPS solutions are extremal.

The paper is organised as follows. In Section 2 we introduce the class of fourdimensional sigma models that we will use for our investigations. Besides the Minkowski signature version two Euclidean versions are introduced, one with a definite, the other with an indefinite action. We review the class of instanton solutions constructed in [26, which are complex saddle points of the definite and real saddle points of the indefinite Euclidean action. In Section 4 we adapt the calculation of semi-classical transition amplitudes of [7, 28, to our class of models and show that a consistent saddle point approximation can be performed using either form of the Euclidean action, and with identical result. In Section 5 we investigate the relation to the third type of Euclidean action, where all axions have been dualized into tensor fields, and point out subtleties related to the treatment of the axionic zero modes. In Section 6 we briefly review how the instanton solutions can be lifted to five-dimensional solitons or black holes, depending on whether the theory is coupled to gravity or not, and compare the instanton action to the soliton and black hole mass. In Section 7 we specialize to supersymmetric models. The Euclidean BPS condition for purely scalar field configurations of $N=2$ vector multiplets is derived and shown to be related to the BPS condition of massive BPS states in one dimension higher. We also find a relation between the BPS condition and the geometry of the target space. We conclude in Section 8 where we identify open questions and future lines of research.

Hilbert space. Thus any problem in one theory can be reformulated within the other. 


\section{Instanton solutions}

\subsection{The Minkowski action}

We start with a theory of $2 n$ scalars $\sigma^{i}, b^{i}, i=1, \ldots, n$ in four-dimensional Minkowski space $M$ with an action of the form

$$
S_{M}[\sigma, b]=-\frac{1}{2} \int_{M} d^{4} x N_{i j}(\sigma)\left(\partial_{\mu} \sigma^{i} \partial^{\mu} \sigma^{j}+\partial_{\mu} b^{i} \partial^{\mu} b^{j}\right)
$$

where $N_{i j}(\sigma)$ is positive definite, and $\mu=0,1,2,3$. This is a non-linear sigma model with $n$ commuting isometries acting as shifts $b^{i} \rightarrow b^{i}+C^{i}$. The corresponding Noether currents and Noether charges are

$$
j_{\mu, i}=N_{i j}(\sigma) \partial_{\mu} b^{j}
$$

and

$$
Q_{i}=\int_{x^{0}=\mathrm{const}} d^{3} \mathbf{x} j_{0, i}(\mathbf{x}, t)
$$

respectively.

The $2 n$ real scalars can be combined into $n$ complex scalar fields

$$
X^{i}=\sigma^{i}+i b^{i},
$$

and the resulting line element on the scalar manifold $\mathcal{M}$ is Hermitean:

$$
d s_{\mathcal{M}}^{2}=N_{i j}(\sigma)\left(d \sigma^{i} d \sigma^{j}+d b^{i} d b^{j}\right)=N_{i j}(X+\bar{X}) d X^{i} d \bar{X}^{j}
$$

Due to the shift symmetries, we can interpret $\mathcal{M}$ as the tangent bundle $T \mathcal{S}$ of the manifold $\mathcal{S}$ parametrized by the scalars $\sigma^{i}$ alone, with line element $d s_{\mathcal{S}}^{2}=$ $N_{i j}(\sigma) d \sigma^{i} d \sigma^{j}$.

An important subclass, where solutions can be obtained explicitly, are manifolds $\mathcal{M}$ which are not only Hermitian, but Kähler. This is equivalent to requiring that $\mathcal{S}$ is Hessian [34]. If $N_{i j}$ is a Hessian metric on $\mathcal{S}$ with Hesse potential $\mathcal{V}(\sigma)$

$$
N_{i j}=\frac{\partial^{2} \mathcal{V}}{\partial \sigma^{i} \partial \sigma^{j}}
$$

then

$$
K(X, \bar{X})=K(X+\bar{X})=4 \mathcal{V}(\sigma(X+\bar{X}))
$$

is a Kähler potential for $d s_{\mathcal{M}}^{2}=N_{i j} d X^{i} d \bar{X}^{j}$ :

$$
N_{i j}=\frac{\partial^{2} K}{\partial X^{i} \partial \bar{X}^{j}}
$$

\subsection{The definite Euclidean action}

The standard version of the Euclidean action is obtained by Wick rotation $x^{0}=-i t$. Here $x^{0}$ denotes Minkowski time and $t$ denotes Euclidean time. The Wick rotation amounts to taking $t$ rather than $x^{0}$ to be real. The Euclidean action is

$$
S_{E}[\sigma, b]=\frac{1}{2} \int_{E} d^{4} x N_{i j}(\sigma)\left(\partial_{m} \sigma^{i} \partial^{m} \sigma^{j}+\partial_{m} b^{i} \partial^{m} b^{j}\right),
$$


where $m=1,2,3,4$ are Euclidean indices. Here $x^{4}=t$ is Euclidean time and if we want to emphasize this interpretation we use $t$ instead of 4 as an index. The Euclidean action is positive definite, $S_{E} \geq 0$, and takes its minimal value $S_{E}=0$ for constant scalars. The partition function

$$
Z=\int D \sigma D b e^{-S_{E}(\sigma, b)}
$$

is 'damped', i.e. fluctuations around saddle points are suppressed, and we expect that the semi-classical approximation (saddle point approximation) is meaningful. But by Derrick's theorem 3, 4 there are no real saddle points. The complex saddle points relevant for instanton effects can be found by using a different type of Euclidean action.

\subsection{The indefinite scalar action}

Let us next discuss another choice for the Euclidean action, where the Wick rotation is combined with an analytical continuation $b^{i} \rightarrow i b^{i}$ of the axionic scalars. To discuss the definite and indefinite version of the Euclidean action in parallel we will use the notation $\beta^{i}=i b^{i}$ for the rotated axionic fields. Both Euclidean actions are related by analytic continuation, and it is useful to consider them as two different real forms of a complex action, which arise by taking either $\beta^{i}$ or $b^{i}$ to be real. The indefinite Euclidean action is

$$
\tilde{S}_{E}[\sigma, \beta]=\frac{1}{2} \int_{E} d^{4} x N_{i j}(\sigma)\left(\partial_{m} \sigma^{i} \partial^{m} \sigma^{j}-\partial_{m} \beta^{i} \partial^{m} \beta^{j}\right) .
$$

The modified scalar manifold $\mathcal{E}$ obtained by rotating the axions has the line element

$$
d s_{\mathcal{E}}^{2}=N_{i j}(\sigma)\left(d \sigma^{i} d \sigma^{j}-d \beta^{i} d \beta^{j}\right) .
$$

This has split signature $(n, n)$ so that the metric and the corresponding action are indefinite. To understand the geometry underlying instanton solutions it is useful to note $\mathcal{E}$ carries a para-Hermitian metric, in analogy to the Hermitian metric on $\mathcal{M}$. In terms of local coordinates, we can combine the $2 n$ real scalar fields $\sigma^{i}$ and $b^{i}$ into $n$ para-complex fields

$$
X^{i}=\sigma^{i}+e \beta^{i}
$$

where the para-complex unit satisfies

$$
e^{2}=1, \quad \bar{e}=-e .
$$

Para-complex geometry is in many respects analogous to complex geometry 4 An almost para-complex structure $J$ is a tensor field of type $(1,1)$ which satisfies

$$
J^{2}=\mathbb{1}
$$

and the additional requirement that $J$ has an equal number of eigenvalues +1 and -15 An almost para-complex structure is called a para-complex structure, if the analogue of the Nijenhuis tensor vanishes, and this condition is equivalent

\footnotetext{
${ }^{4}$ See [20] for a comprehensive review.

${ }^{5}$ If the second requirement is dropped, then $J$ is an almost product structure.
} 
to the existence of local para-complex coordinates. If a line element takes the form

$$
d s_{\mathcal{E}}^{2}=N_{i j} d X^{i} d \bar{X}^{j}
$$

then it is para-Hermitian 6 Moreover, if $N_{i j}$ is a Hessian metric on $\mathcal{S}$, then $K(X, \bar{X})=4 \mathcal{V}(\sigma)$ is a para-Kähler potential and $\mathcal{E}$ is a para-Kähler manifold. Like $\mathcal{M}$ we can interprete $\mathcal{E}$ as the tangent bundle of $\mathcal{S}$, but equipped with a different metric.

Since the line element of $\mathcal{E}$ and the corresponding Euclidean action are indefinite, Derrick's theorem does not apply and it is possible to find solutions of the equations of motion

$$
\begin{aligned}
\partial^{m}\left(N_{i j} \partial_{m} \sigma^{j}\right)-\frac{1}{2} \partial_{i} N_{j k}\left(\partial_{m} \sigma^{i} \partial^{m} \sigma^{j}-\partial_{m} \beta^{i} \partial^{m} \beta^{j}\right) & =0, \\
\partial^{m}\left(N_{i j} \partial_{m} \beta^{j}\right) & =0 .
\end{aligned}
$$

A particular class, which we call extremal instanton solutions, is obtained by imposing the ansatz

$$
\partial_{m} \sigma^{i}= \pm \partial_{m} \beta^{i} .
$$

We will see later that this ansatz is indeed related to the saturation of a Bogomol'nyi bound, and that it is satisfied by $\frac{1}{2}$ BPS solutions for Euclidean vector multiplets. Moreover, it implies that extremal instanton solutions remain unmodified when coupling the sigma model to gravity, and that one can obtain extremal black hole solution by dimensional lifting with respect to time. For the time being we observe that the extremal instanton ansatz leads to an enormous simplification of the equations of motion, which now reduce to

$$
\partial^{m}\left(N_{i j} \partial_{m} \sigma^{j}\right)=0
$$

This simplification can be understood in terms of the para-complex geometry of the scalar target space $\mathcal{E}$. Geometrically, the equations of motion for a nonlinear sigma model are the equations for a harmonic map from space-time into the scalar target space. One way to obtain harmonic maps is to find harmonic maps into completely geodesic submanifolds 1, 2, 20. The extremal instanton ansatz (4) imposes that tangent vectors to solutions are null vectors and restricts the solution to take values in a totally isotropic submanifold. Totally isotropic submanifolds are known to be intimately related to extremal solutions, but it remains to show that the submanifolds defined by (4) are totally geodesic. But this is automatic if we assume that $\mathcal{S}$ is Hessian, so that $\mathcal{E}$ is para-Kähler [20]. The extremal instanton ansatz implies that the tangent vectors of the solution lie within the eigenspaces of the para-complex structure, and for a para-Kähler manifold the para-complex structure is parallel. Therefore the corresponding submanifolds are totally geodesic. We can in fact obtain the solution explicitly. Using that $N_{i j}$ has a Hesse potential $\mathcal{V}(\sigma)$ we can define dual scalar fields

$$
\sigma_{i}=\partial_{i} \mathcal{V}
$$

\footnotetext{
${ }^{6}$ To be able to define a fundamental two-form, the para-complex structure is required to act as an anti-isometry. In the context of 'doubled geometries', this property is sometimes referred to as 'pseudo-Hermitian' 30]. However, in the mathematical literature pseudo-Hermitian usually refers to complex structures which are isometries of indefinite metrics.
} 
and the equations of motion reduce to harmonic equations

$$
\Delta \sigma_{i}=0 .
$$

Therefore the solution depends on $n$ harmonic functions $H_{i}(x)$. Note that while $\left\{\sigma^{i}, b^{i}\right\}$ are uniquely determined (up to integration constants for the $b^{i}$ to be discussed later) in terms of the harmonic functions, we can not always solve (5) explicitly for the original scalars $\sigma^{i}$. The equations (5) are a set of coupled algebraic equations, which, by dimensional lifting over time, are related to the five-dimensional black hole attractor equations [26. We note that the this type of solution relies on the indefinite signature of the scalar manifold. For definite signature we would only have found constant maps, i.e. constant scalar fields corresponding to ground state solutions rather than instanton solutions.

We also observe that if we substitute the instanton solution $\left\{\sigma_{*}^{i}, \beta_{*}^{i}\right\}$ back into the action, we obtain zero

$$
\tilde{S}_{E}\left[\sigma_{*}, \beta_{*}\right]=0 .
$$

This is in fact an automatic consequence of the ansatz (4), and thus a flip side of precisely the feature which allows the existence of non-trivial saddle points in the first place. We will see later that a finite and positive instanton action is obtained by adding a boundary term.

\subsection{Complex saddle points}

The definite and the indefinite Euclidean action are related by analytical continuation of the axions. Introducing complex axion fields

$$
B^{i}=b^{i}+i \beta^{i},
$$

the two actions can be obtained as two different 'real forms' of the complex action

$$
S_{E}[\sigma, B]=\frac{1}{2} \int_{E} d^{4} x N_{i j}(\sigma)\left(\partial_{m} \sigma^{i} \partial^{m} \sigma^{j}+\partial_{m} B^{i} \partial^{m} B^{j}\right) .
$$

The scalar target space parametrized $\left\{\sigma^{i}, B^{i}=b^{i}+i \beta^{i}\right\}$ can be interpreted as the complexified tangent bundle $T \mathcal{S}_{\mathbb{C}}$ of $\mathcal{S}$. Note that $d s^{2}=N_{i j}(\sigma) d B^{i} d B^{j}$ is a complex bilinear form (and not a Hermitian sesquilinear form) on the tangent spaces $T_{P} \mathcal{S}$.

It is clear that real saddle points of the indefinite action (3) can be interpreted as complex (purely imaginary) saddle points of the definite action (2). Thus the first two of the ways around Derrick's Theorem mentioned in the introduction, complex saddle points and indefinite actions, are equivalent for this class of models. However, this does not address the problem that the instanton action is identically zero, and since we are lead to considering complex field values the issue of the damping of the functional integral also deserves further investigation.

\section{Instanton amplitudes}

In the context of quantum physics, we are interested in instantons because non-trivial saddle points of the Euclidean functional integral give rise to nonperturbative corrections. To see whether the solutions found above can play 
such a role, we need to consider suitable physical quantities, the two obvious candidates being the partition function and transition amplitudes between states of different axionic charge 7

We find it helpful to exploit the analogy with the saddle point approximation of one-dimensional real integrals, which often uses complex variables and contour deformation. As we will demonstrate the 'complex viewpoint' leads to a transparent and unified treatment of instantons for axionic scalars, their dual description in terms of tensor fields, and their lifting to solitonic solutions, including black holes.

\subsection{A toy example for the saddle point approximation}

The problem of finding a consistent saddle point approximation can be illustrated with a simple, one-dimensional toy example. We consider an integral over the real line

$$
I=\int_{-\infty}^{\infty} d x e^{-f(x)}
$$

which is meant to serve as a toy example either for the 'axionic' partition function

$$
Z=\int D b e^{-S_{E}[b]},
$$

or for any amplitude that we might want to compute 8 Let us assume that $f(x)$ can be continued analytically into the complex $z$ plane, $z=x+i y$, and that it has a sharp saddle point at $z_{*}=i \alpha$, where $\alpha$ is real. Then the saddle point approximation is obtained by performing a Gaussian integration over the saddle point. For concreteness (and since it will fit with the functional integrals we are interested in) we further assume that $f(x)$ has a minimum if we pass through the stationary point on a contour parallel to the real axis:

$$
\partial_{x}^{2} f(z)_{z=z_{*}}>0
$$

The saddle point approximation is obtained by expanding $f$ to second order and taking the integration contour to be $z(x)=x+i \alpha$, where $-\infty<x<\infty$ :

$$
\begin{aligned}
I & \approx \int_{-\infty+i \alpha}^{\infty+i \alpha} d z e^{-f\left(z_{*}\right)-\frac{1}{2} f^{\prime \prime}\left(z_{*}\right)\left(z-z_{*}\right)^{2}}=\int_{-\infty}^{\infty} d x e^{-f(i \alpha)-\frac{1}{2} \partial_{x}^{2} f(i \alpha) x^{2}} \\
& \simeq\left(\partial_{x}^{2} f(i \alpha)\right)^{-1 / 2} e^{-f(i \alpha)}
\end{aligned}
$$

In the analogy, $f(i \alpha)$ is the 'instanton action', which should be positive, finite and non-vanishing, $\partial_{x}^{2} f(i \alpha)>0$ is the 'fluctuation determinant', which needs to be positive definite so that the Gaussian integral is damped and the saddle point approximation is consistent. In the following we will verify that the toy example indeed captures the essential properties of describing instantons from the viewpoint of the definite Euclidean action.

To obtain an analogue for the situation concerning the indefinite Euclidean action, we compute the same integral using a new, rotated complex variable

\footnotetext{
${ }^{7}$ As we will see axionic charges play a role analogous to instanton charges in Yang-Mills theories, and therefore we will refer to them sometimes as instanton charges.

${ }^{8}$ We suppress the scalars $\sigma^{i}$ since the issue we are interested in is the treatment of the axions.
} 
$w=-i z$. Note that this is the correct analogy, because we are not interested in modifying the physical, Minkowski signature theory, but just use a different Euclidean continuation to compute the same physical quantity. In terms of the rotated variable the function $g(w)=f(z(w))$ now has a real saddle point at $w_{*}=\alpha$, and since fluctuations of $f$ around $z_{*}$ were damped along the real axis, fluctuations of $g$ around $w_{*}$ are damped along the imaginary axis, i.e. (7) is equivalent to

$$
\partial_{v}^{2} g\left(w_{*}\right)>0
$$

where $w=u+i v$. In terms of the rotated variable the integral $I$ takes the form

$$
I=-i \int_{i \infty}^{-i \infty} d w e^{-g(w)}
$$

and the saddle point approximation takes the form

$$
\begin{aligned}
I & \approx-i \int_{i \infty+\alpha}^{-i \infty+\alpha} d w e^{-g\left(w_{*}\right)-\frac{1}{2} g^{\prime \prime}\left(w_{*}\right)\left(w-w_{*}\right)^{2}}=\int_{-\infty}^{\infty} d v e^{-g(\alpha)-\frac{1}{2} \partial_{v}^{2} g(\alpha) v^{2}} \\
& \simeq\left(\partial_{v}^{2} g(\alpha)\right)^{-1 / 2} e^{-g(\alpha)} .
\end{aligned}
$$

We will now demonstrate that this is the correct analogy for the use of the two Euclidean actions for the computation of instanton amplitudes.

\subsection{Instantons from the Wick rotated scalar action}

In this section we adapt a classical computation done in [7] for axionic wormholes to our type of models. We closely follow the presentation given by [28] for hypermultiplet instantons. The amplitude to be computed is the transition amplitude between two different field configurations of the axions, which are specified on spatial hyperplanes. We will treat the $\sigma^{i}$ as spectators in the following, and suppress them in most of the formulae. Let us denote the initial and final field configurations of the fields $b^{i}(x)$ by $\chi_{I}^{i}(\mathbf{x})$ and $\chi_{F}^{i}(\mathbf{x})$, respectively. Here and in the following $x$ are coordinates on Euclidean space $E=\mathbb{R}_{t} \times$ $\mathbb{R}^{3}, t$ is Euclidean time and $\mathbf{x}$ are coordinates on space $\mathbb{R}^{3}$. The asymptotic configurations are imposed at the times $t_{I}$ and $t_{F}$, which we ultimately take to the limits $t_{I} \rightarrow-\infty$ and $t_{F} \rightarrow \infty$.

We are interested in computing the quantum transition amplitude between the initial state $|I\rangle=\left|\chi_{I}\right\rangle$ and the final state $|F\rangle=\left|\chi_{F}\right\rangle$. Here $|I\rangle=\left|\chi_{I}\right\rangle=$ $\left|\chi_{I}^{i}(\mathbf{x})\right\rangle$ is a formal 'position' eigenstate, where the fields $b^{i}$ assume the configuration $\chi_{I}^{i}(\mathbf{x})$. The corresponding Euclidean transition amplitude between the two states is

$$
\mathcal{A}=\left\langle F\left|e^{-H\left(t_{F}-t_{I}\right)}\right| I\right\rangle,
$$

where $H$ is the Hamiltonian. In the functional formulation this becomes

$$
\mathcal{A}=\int_{B C} D b e^{-S_{E}[b]},
$$

where the fields $b^{i}(x)$ are subject to the boundary conditions $b^{i}\left(\mathbf{x}, t_{I}\right)=\chi_{I}^{i}(\mathbf{x})$ and $b^{i}\left(\mathbf{x}, t_{F}\right)=\chi_{F}^{i}(\mathbf{x})$. As announced, we suppress the fields $\sigma^{i}$ for the time being. 
As usual in quantum mechanics and quantum field theory, we are interested in computing transition amplitudes for asymptotically large time. The resulting asymptotic transition amplitudes are time-independent, and there is no need to explicitly continue back the result from Euclidean to physical time, in contrast to Greens functions. In the limit of asympotically large time only the lowest eigenvalue of the Hamiltonian contributes. While naively this would restrict one to compute vacuum amplitudes, one can also access amplitudes between the ground states of charge (superselection) sectors by inserting suitable projection operators [7. In our case the theory has $n$ conserved charges related to the $n$ commuting shift symmetries, and we can ask for transition amplitudes between states with fixed initial and final axionic charges $Q_{i}^{I / F}$. Since states are characterized by specifying field configurations rather than global (integrated) quantities, the relevant quantity to compute is the transition amplitude between states with prescribed initial and finite charge densities $\rho_{i}^{I / F}(\mathbf{x})$, where $Q_{i}^{I / F}=\int_{I / F} d^{3} \mathbf{x} \rho_{i}^{I / F}(\mathbf{x})$. The projection of the amplitude $\mathcal{A}$ onto such states amounts to the insertion of a functional delta function into the amplitude, which forces the time-like components of the Noether currents $j_{m \mid i}(x)$ to take prescribed values at $t_{I}$ and $t_{F}$. Specifically, for the initial state we have

$$
P_{I}|I\rangle=\delta\left(\rho^{I}-j_{t}^{I}\right)\left|\chi_{I}\right\rangle,
$$

where $j_{t}^{I}=j_{t \mid i}^{I}(\mathbf{x})=j_{t \mid i}\left(\mathbf{x}, t_{I}\right)$ is the Noether current at time $t=t_{I}$, and the delta function is a formal 'functional delta function'

$$
\delta\left(\rho^{I}-j_{t}^{I}\right)=\prod_{\mathbf{x} \in \mathbb{R}_{t=t_{I}}^{3}} \prod_{i=1}^{n} \delta\left(\rho_{i}^{I}(\mathbf{x})-j_{t \mid i}^{I}(\mathbf{x})\right),
$$

which imposes the boundary values for the Noether current.

In order to implement this projection in the functional integral, we use the Fourier representation of the delta function. For a functional delta function this leads to an additional functional integral over auxiliary functions $\gamma_{I}=\left(\gamma_{I}^{i}(\mathbf{x})\right)$ and $\gamma_{F}=\left(\gamma_{F}^{i}(\mathbf{x})\right)$ which 'live' on the initial and final hypersurface:

$$
P_{I}|I\rangle=\int_{I} D \gamma_{I} e^{-i \int_{I} d^{3} \mathbf{x}\left(\rho^{I}-j_{t}^{I}\right) \cdot \gamma_{I}}\left|\chi_{I}\right\rangle
$$

To avoid cluttered formulae we use the letters $I$ and $F$ to indicate that an integral is taken over the initial or final hypersurface, or that a functional integral is taken over functions on the initial and final hypersurface. The arguments $x, \mathbf{x}$ are omitted where possible without ambiguity. We use the short hand notation

$$
\left(\rho^{I}-j_{t}^{I}\right) \cdot \gamma_{I}=\left(\rho_{i}^{I}(\mathbf{x})-j_{t \mid i}^{I}(\mathbf{x})\right) \gamma_{I}^{i}(\mathbf{x}) .
$$

We now use that the Noether charges $Q_{i}$ generate shift symmetries $b^{i} \rightarrow b^{i}+C^{i}$. Since $j_{m \mid i}$ is the corresponding Noether current, the combination $j_{t}^{I} \cdot \gamma_{I}$ generates shifts with (local) parameter $\gamma_{I}^{i}(\mathbf{x})$, and acts on states as $\left|\chi_{I}\right\rangle \rightarrow\left|\chi_{I}+\gamma_{I}\right\rangle$. Therefore

and similarly

$$
P_{I}|I\rangle=\int_{I} D \gamma_{I} e^{-i \int_{I} d^{3} \mathbf{x} \rho^{I} \cdot \gamma_{I}}\left|\chi_{I}+\gamma_{I}\right\rangle
$$

$$
P_{F}|F\rangle=\int_{F} D \gamma_{F} e^{-i \int_{F} d^{3} \mathbf{x} \rho^{F} \cdot \gamma_{F}}\left|\chi_{F}+\gamma_{F}\right\rangle .
$$


The charge density projected amplitude is

$$
\begin{aligned}
& \tilde{\mathcal{A}}=\left\langle F\left|P_{F} e^{-H\left(t_{F}-t_{I}\right)} P_{I}\right| I\right\rangle \\
= & \int_{F} D \gamma_{F} \int_{I} D \gamma_{I} \int_{B C} D b e^{i \int_{F} d^{3} \mathbf{x} \rho^{F} \cdot \gamma_{F}} e^{-i \int_{I} d^{3} \mathbf{x} \rho^{I} \cdot \gamma_{I}} e^{-S_{E}[b]},
\end{aligned}
$$

with boundary conditions

$$
b^{i}\left(\mathbf{x}, t_{I}\right)=\chi_{I}^{i}(\mathbf{x})+\gamma_{I}^{i}(\mathbf{x}), \quad b^{i}\left(\mathbf{x}, t_{F}\right)=\chi_{F}^{i}(\mathbf{x})+\gamma_{F}^{i}(\mathbf{x})
$$

The three functional integrals can be combined into a single integral over fields $b^{i}(x)$ without boundary conditions. Setting

$$
\gamma_{I / F}=\tilde{\gamma}_{I / F}-\chi_{I / F},
$$

the amplitude becomes

$$
\tilde{\mathcal{A}}=\int_{F} D \tilde{\gamma}_{F} \int_{I} D \tilde{\gamma}_{I} \int_{B C} D b e^{i \int_{F} d^{3} \mathbf{x}\left(\tilde{\gamma}_{F}-\chi_{F}\right) \cdot \rho^{F}} e^{-i \int_{I} d^{3} \mathbf{x}\left(\tilde{\gamma}_{I}-\chi_{I}\right) \cdot \rho^{I}} e^{-S_{E}[b]},
$$

with boundary conditions

$$
b^{i}\left(\mathbf{x}, t_{I / F}\right)=\tilde{\gamma}_{I / F}^{i}(\mathbf{x}) .
$$

The functional integrals over $\tilde{\gamma}_{I / F}$ are now integrations over boundary conditions for the $b^{i}(x)$, so that we obtain an integral over $b^{i}(x)$ without boundary conditions,

$$
\tilde{\mathcal{A}}=e^{-i \int_{F} \chi_{F} \cdot \rho^{F}+i \int_{I} \chi_{I} \cdot \rho^{I}} \int D b e^{i \int_{F} b^{i} \rho_{i}^{F}-i \int_{I} b^{i} \rho_{i}^{I}} e^{-S_{E}[b]} .
$$

The phase factor in front of the remaining functional integral depends on the field configurations $\chi_{I / F}$ and charge densities $\rho^{I / F}$ which define the initial and final states $P_{I / F}|I / F\rangle$. This pre-factor keeps track of the relation between the 'position eigenstates' and the 'charge eigenstates'. The remaining functional integral is a transition amplitude between charge (density) eigenstates. While the integration over the fields $b^{i}$ is now unrestricted, this integral is still sensitive to the boundary conditions, which encode the physical states, through the boundary term. As we will see this boundary term determines the saddle point which dominates the saddle point approximation. If the boundary term is dropped, the remaining functional integral is simply the partition function. As we will see the same boundary term also occurs in various other contexts (dimensional lifting and Hodge dualization), so that it is natural to regard $S_{E}+\Sigma$ as a modified or 'improved' action. For the amplitude at hand the boundaries which support the boundary term are spatial hyperplanes located at $t=t_{I}$ and $t=t_{F}$, respectively. The two boundary terms can be combined into the more compact expression

$$
\Sigma=i \int_{I} d^{3} \mathbf{x} b^{i} \rho_{i}^{I}-i \int_{F} d^{3} \mathbf{x} b^{i} \rho_{i}^{F}=-i \oint_{\partial E} b^{i} \rho_{i}
$$

where $\partial E$ is the combined boundary, with the orientation chosen such that the future directed normal is the outer normal 9 We remark in passing that the last

${ }^{9}$ Thus in terms of homology classes, $[\partial E]=[F]-[I]$. 
formula can be used when other types of boundaries are relevant. For example, for spherically symmetric instanton solutions, such as hypermultiplet or vector multiplet instantons, the boundary is an asymptotic three sphere, $\partial E=S_{(\infty)}^{3}$. As we will see later such instanton solutions can be lifted to black hole solutions.

Let us summarize our result for the amplitude

$$
\tilde{\mathcal{A}}=\tilde{\mathcal{A}}\left(\chi_{I}, \chi_{F}, \rho^{I}, \rho^{F}\right)=e^{-i \oint \chi \cdot \rho} \int D b e^{-S_{E}[b]-\Sigma[b \mid \rho]},
$$

before proceeding to the saddle point approximation. When identifying critical points of the action, we have to take into account the boundary term. We consider 'boundary variations' and 'bulk variations' separately, starting with the boundary variations. Here two contributions arise. The obvious one is the variation of the boundary term:

$$
\delta \Sigma=-i \oint d^{3} \mathbf{x} \delta b^{i} \rho_{i}
$$

But boundary terms also arise from variations of the bulk action upon integration by parts.

$$
\begin{aligned}
\delta S & =\int d^{4} x N_{i j}(\sigma) \partial_{m} \delta b^{i} \partial^{m} b^{j} \\
& =\int d^{4} x \partial_{m}\left(N_{i j}(\sigma) \partial^{m} b^{j} \delta b^{i}\right)-\int d^{4} x \partial_{m}\left(N_{i j}(\sigma) \partial^{m} b^{j}\right) \delta b^{i} \\
& =\oint d^{3} \mathbf{x} n^{m} N_{i j}(\sigma) \partial_{m} b^{j} \delta b^{i}-\int d^{4} x \partial_{m}\left(N_{i j}(\sigma) \partial^{m} b^{j}\right) \delta b^{i}
\end{aligned}
$$

Here $n^{m}$ is the outer unit normal vector of the boundary. Note that this vector points into the positive direction on the final hypersurface and into the negative direction on the initial hypersurface. The second term gives rise to the equation of motion of the $b^{i}$,

$$
\partial_{m}\left(N_{i j}(\sigma) \partial^{m} b^{j}\right)=0,
$$

which expresses conservation of the current associated with the shift symmetry. The first term contributes to the variation at the boundary, and the resulting total boundary variation is

$$
\begin{aligned}
(\delta S+\delta \Sigma)_{\text {boundary }} & =\oint\left(n^{m} N_{i j}(\sigma) \partial_{m} b^{j} \delta b^{i}-i \delta b^{i} \rho_{i}\right) \\
& =\int_{F} d^{3} \mathbf{x}\left(N_{i j} \sigma \partial_{t} b^{j}-i \rho_{i}^{F}\right) \delta b^{i}-\int_{I} d^{3} \mathbf{x}\left(N_{i j} \sigma \partial_{t} b^{j}-i \rho_{i}^{I}\right) \delta b^{i} .
\end{aligned}
$$

Vanishing of the boundary variation implies

$$
\begin{aligned}
\left(N_{i j}(\sigma) \partial_{t} b^{j}\right)_{t=t_{I}} & =i \rho_{i}^{I}(\mathbf{x}) \\
\left(N_{i j}(\sigma) \partial_{t} b^{j}\right)_{t=t_{F}} & =i \rho_{i}^{F}(\mathbf{x}) .
\end{aligned}
$$

The factors $i$ might at first appear surprising. The functions $\rho_{i}^{I / F}(\mathbf{x})$ are the charge densities of the physical states entering our amplitude and must therefore be real, and thus the saddle point solution for the $b^{i}$ must satisfy imaginary boundary conditions. While this might be counter-intuitive, these boundary 
conditions are physically meaningful, because, following [7], we have projected onto eigenstates of the Euclidean Noether current. The Wick rotation introduces an a factor of $i$ in the time component of vectors, and if we write the boundary condition in terms of Minkowski time, we obtain

$$
\left(N_{i j}(\sigma) \partial_{0} b^{j}\right)_{I / F}=\rho_{i}^{I / F}
$$

Moreover, inspection of the bulk variation shows that the saddle point solution which dominates the Euclidean functional integral is indeed imaginary.

In order to find explicit and non-trivial saddle points we need to re-instate the scalar fields $\sigma^{i}$, which we have neglected so far. Then the full bulk action is

$$
S_{E}[\sigma, b]=\frac{1}{2} \int d^{4} x N_{i j}(\sigma)\left(\partial_{m} \sigma^{i} \partial^{m} \sigma^{j}+\partial_{m} b^{i} \partial^{m} b^{j}\right) .
$$

The bulk variation of $b^{i}$ is not modified. The variation of the $\sigma^{i}$ gives their equation of motion

$$
\partial^{m}\left(N_{i j}(\sigma) \partial_{m} \sigma^{j}\right)-\frac{1}{2} \partial_{i} N_{j k}(\sigma)\left(\partial_{m} \sigma^{j} \partial^{m} \sigma^{k}+\partial_{m} b^{j} \partial^{m} b^{k}\right)=0
$$

The extremal instanton solutions discussed previously are obtained by imposing the extremal instanton ansatz

$$
\partial_{m} \sigma^{i}= \pm i \partial_{m} b^{i}
$$

Since we are working with the definite Euclidean action, the saddle point solution of the bulk action $b_{*}^{i}$ is imaginary. This is consistent with the imaginary boundary conditions which we found from the boundary variation, and therefore the saddle point contributes to the amplitude we are computing.

Let us therefore turn to the saddle point evaluation of the amplitude $\tilde{\mathcal{A}}$. We denote the saddle point solution by $\sigma_{*}^{i}$ and $b_{*}^{i}=i \beta_{*}^{i}$, where $\beta_{*}^{i}$ is real and satisfies the boundary condtions

$$
\left(N_{i j}(\sigma) \partial_{t} \beta^{j}\right)_{* \mid I / F}=\rho_{i}^{I / F}
$$

Since we have only specified initial and final states of $b^{i}$, and not of $\sigma^{i}$, there are no boundary conditions for the $\sigma^{i}$. We only impose that they satisfy their bulk equations of motion, and this will lead to a consistent semi-classical transition amplitude for the $b^{i}$. Thus the $\sigma^{i}$ are treated as classical on shell background fields.

The leading contribution to the quantum amplitude $\tilde{A}$ comes from evaluating the action at the saddle point. As we have already noted previously, the bulk action vanishes at the saddle point:

$$
S_{E}\left[\sigma_{*}, b_{*}\right]=\frac{1}{2} d^{4} x \int N_{i j}\left(\sigma_{*}\right)\left(\partial_{m} \sigma_{*}^{i} \partial^{m} \sigma_{*}^{j}+\partial_{m} b_{*}^{i} \partial^{m} b_{*}^{j}\right)=0,
$$

as a consequence of the ansatz (4). However we now see that the day is saved by the boundary action, which gives

$$
\Sigma\left[b_{*}\right]=-i \oint d^{3} \mathbf{x} b_{*}^{i} \rho_{i}=\oint d^{3} \mathbf{x} \beta_{*}^{i} \rho_{i}=\beta_{*}^{i}\left(t_{F}\right) Q_{i}^{F}-\beta_{*}^{i}\left(t_{I}\right) Q_{i}^{I},
$$


and therefore

$$
\tilde{\mathcal{A}} \propto e^{-S_{*}-\Sigma_{*}}=e^{-\Sigma_{*}}=e^{-\beta^{i}\left(t_{F}\right) Q_{i}^{F}-\beta^{i}\left(t_{I}\right) Q_{i}^{I}} .
$$

In particular, if $\beta_{*}^{i}\left(t_{F}\right)=\beta_{*}^{i}\left(t_{I}\right)=\beta_{0}$, then $\Sigma_{*}=\beta_{0}\left(Q_{i}^{F}-Q_{i}^{I}\right)$. Thus the instanton amplitude is proportional to the difference between the charges of the initial and final state, as expected for a tunneling amplitude between ground states of charge (superselection) sectors. We remark that in contrast to the classical action the transition amplitude depends on the values $\beta_{*}^{I}\left(t_{F / I}\right)$ of the axions. The classical continuous shift symmetry is broken to a discrete subset of imaginary shifts in $\beta^{i}$ and thus real shifts in $b^{i}$ :

$$
\beta^{i} \rightarrow \beta^{i}+2 \pi i k \Leftrightarrow b^{i} \rightarrow b^{i}+2 \pi k, \quad k \in \mathbb{Z} .
$$

The breaking of continuous shift symmetries by instantons is a typical quantum effect. Since the shift symmetry is a global symmetry it does not lead to an inconsistency of the theory.

Finally, a consistent saddle point approximation requires that the functional integral is damped, i.e. that the fluctuation determinant is positive definite. This is indeed the case, as long as the functional integration is performed along 'real directions' in $b^{i}$-field space. More precisely, when shifting the integration variable according to

$$
b^{i}=i \beta_{*}^{i}+\tilde{b}^{i},
$$

where $\beta_{*}^{i}$ is the saddle point solution, and where the (real) fluctuation $\tilde{b}^{i}$ is the new integration variable, then

$$
\tilde{\mathcal{A}} \propto e^{-\Sigma_{*}} \int D \tilde{b} e^{-\frac{1}{2} \int d^{4} x N_{i j}\left(\sigma_{*}\right) \partial_{m} \tilde{b}^{i} \partial^{m} \tilde{b}^{j}}
$$

Since $N_{i j}$ is assumed to be positive definite, the fluctuation determinant is damped and leads to a Gaussian integral. Therefore the saddle point approximation is well defined. Comparing (15) to the one-dimensional toy example we see that this indeed captures the essential features of the saddle point approximation of the axionic functional integral.

\subsection{Instantons from the indefinite scalar Euclidean action}

Let us now address the issue whether the indefinite action (3) can play a role in computing instanton amplitudes, despite that it is not positive definite. Here the key point is to note that irrespective of how we define an (or 'the') Euclidean version of the theory, we do not intend to change the physical, Minkowski signature theory. Therefore we still want to compute the same physical amplitude as before, albeit using a different analytical continuation. The initial and final states of the amplitude are 'position' or 'charge' eigenstates of the original Minkowski signature field $b^{i}$. Thus when using the rotated axion field $\beta^{i}=-i b^{i}$, the boundary condition of the functional integral which encodes the initial and final state are

$$
i \beta^{i}\left(\mathbf{x}, t_{I / F}\right)=\chi_{I / F}^{i}(\mathbf{x}) .
$$

While within our approach it is clear from the start that we are just rewriting the amplitude in terms of a different, rotated integration variable, it is instructive 
to revisit some of the key formulae of the previous section and to express them in terms of the variable $\beta^{i}$. We already saw that the variation of the bulk action $\tilde{S}_{E}$ with respect to the rotated axions $\beta^{i}$ leads to a real saddle point $\beta^{i}=\beta_{*}^{i}$. However the boundary conditions (16) of the functional integral over $\beta^{i}$ are now imaginary 10 The charge projected amplitude can be brought to the form

$$
\tilde{A}=e^{-i \oint \chi \cdot \rho} \int D \beta e^{-\tilde{S}_{E}[\beta]-\tilde{\Sigma}[\beta \mid \rho]} .
$$

The prefactor is the same as before, since $\chi$ and $\rho$ characterize physical position and charge eigenstates and are not subject to analytical continuation. In the saddle point approximation the $\beta^{i}$ decompose into a real saddle point solution $\beta_{*}^{i}$ and purely imaginary fluctuations $\tilde{\beta}^{i}=-i \tilde{b}^{i}$ :

$$
\beta^{i}=\beta_{*}^{i}+\tilde{\beta}^{i}=\beta_{*}^{i}-i \tilde{b}^{i} .
$$

As in the rotated version of the one-dimensional toy example the integration, the original integration is over purely imaginary field configurations ('integration along the imaginary axis'), and get shifted by the real saddle point solution.

The boundary term is

$$
\tilde{\Sigma}=\oint d^{3} \mathbf{x} \beta^{i} \rho_{i}
$$

and the vanishing of the total boundary variation (variation of boundary terms plus terms obtained by integration by parts) implies

$$
\left(N_{i j}(\sigma) \partial_{t} \beta^{j}\right)_{I / F}=\rho_{i}^{I / F}
$$

which is consistent with a real saddle point solution. Also note that the Euclidean Noether current for the indefinite action is indeed real, because both time and the axion field have been Wick rotated.

The integration over fluctuations takes the form

$$
\int D \tilde{\beta} e^{\frac{1}{2} \int d^{4} x N_{i j} \partial_{m} \tilde{\beta}^{i} \partial^{i} \tilde{\beta}^{j}}
$$

This is damped for positive definite $N_{i j}$ if the fluctuations are purely imaginary. This is consistent with the imaginary boundary conditions of the original integral that we approximate.

So far we have insisted that the two Euclidean actions we compare are Euclidean versions of the same Minkowski signature theory. Alternatively, one might take the viewpoint that the Euclidean theory is to be taken fundamental. In this approach a theory is defined by its Euclidean functional integral, and physical quantities in Minkowski space are obtained by analytical continuation in either position or momentum space. From this point of view it is conceivable that physically inequivalent Euclidean actions could be found. But for the class of actions considered here it is clear as a result of the above analysis that the requirement of a consistent saddle point approximation does only admit one consistent choice, modulo a choice of variables. We remark that the situation seems to become more complicated if sigma models with a more complicated,

\footnotetext{
${ }^{10} \mathrm{We}$ are referring to the boundary conditions of the functional integral representing the amplitude, not the boundary conditions satisfied by the saddle point solution.
} 
non-Abelian isometry group are considered. In particular, as observed in 28, it can happen that some of the saddle points of the combined bulk and boundary action do not have a real positive instanton action. These complications are also reflected by the observation that the universal hypermultiplet admits that there exist three different real Euclidean versions, one with a definite and two with an indefinite target space, which are different real sections of one underlying complex action [14, 40, 41. Thus once the isometry group is not required to be Abelian, the question which complex saddle points contribute in the semi-classical approximation becomes more complicated, and there are more Euclidean actions to be considered. We plan to investigate actions with non-Abelian isometries in the future, based on generalized version of the c-map.

\section{Instantons from the Euclidean scalar-tensor action}

We now turn to the third Euclidean formulation of the theory, where the axionic scalars $b^{i}$ are replaced by antisymmetric tensor fields $B_{m n \mid i}$. Aspects of this dualization for the class of models under consideration were discussed in detail in 26. Therefore we start with a brief summary of the points relevant for the present discussion, and then add further remarks concerning the relation between the partition functions and the role of axionic zero modes.

The field strength of the antisymmetric tensor fields are

$$
H_{m n p \mid i}=3 ! \partial_{[m} B_{n p] \mid i} \propto N_{i j}(\sigma) \epsilon_{m n p q} \partial^{q} b^{j} .
$$

The Euclidean action of the scalar-tensor theory

$$
S_{E}[\sigma, B]=\int d^{4} x\left(\frac{1}{2} N_{i j}(\sigma) \partial_{m} \sigma^{i} \partial^{m} \sigma^{j}+\frac{1}{2 \cdot 3 !} N^{i j}(\sigma) H_{m n p \mid i} H_{j}^{m n p}\right),
$$

is obtained from its Minkowski counter part by the standard Wick rotation. This Euclidean action is positive definite, and a Bogomol'nyi bound is found by re-writing it as a sum of squares, plus a remainder:

$S_{E}[\sigma, B]=\int d^{4} x\left[\frac{1}{2}\left(\partial_{m} \sigma^{i} \mp \frac{1}{3 !} N^{i j}(\sigma) \epsilon_{m n p q} H_{j}^{n p q}\right)^{2} \pm \frac{1}{3 !} \partial_{m} \sigma^{i} \epsilon^{m n p q} H_{n p q \mid i}\right]$.

Thus the action is minimized by imposing

$$
\partial_{m} \sigma^{i}= \pm \frac{1}{3 !} N^{i j}(\sigma) \epsilon_{m n p q} H_{j}^{n p q}
$$

which is the Hodge-dual version of the extremal instanton ansatz (44). After imposing this ansatz, the remaining scalar equations of motion for the $\sigma^{i}$ are the same as in the scalar formulation of the theory, and can be solved in terms of harmonic functions by passing to dual scalars. Substituting the solution back into the action, one obtains

$$
S_{\text {inst }}=\left.\int d^{4} x N_{i j}(\sigma) \partial_{m} \sigma^{i} \partial^{m} \sigma^{j}\right|_{*}
$$


where the $\sigma_{*}^{i}$ are obtained by solving (5) in terms of harmonic functions. While resulting from the bulk action (17) this is a boundary term, modulo the equations of motion:

$$
S_{\mathrm{inst}}=\left.\oint d^{3} \mathbf{x} n^{m} N_{i j}(\sigma) \sigma^{i} \partial_{m} \sigma^{j}\right|_{*} .
$$

The magnetic charges with respect to the $B$-fields are

$$
Q_{i}=\frac{1}{3 !} \oint d^{3} \mathbf{x} n^{m} \epsilon_{m n p q} H_{i}^{n p q},
$$

where we choose the normalization such that they are equal to the electric charges with respect to the $b$-fields. When evaluated on instanton solutions we can use (18) to rewrite this expression as

$$
Q_{i}=\left.\oint d^{3} \mathbf{x} n^{m} N_{i j}(\sigma) \partial_{m} \sigma^{j}\right|_{*} .
$$

The resulting instanton action is

$$
S_{\text {inst }}=\sigma^{i}\left(t_{F}\right) Q_{i}^{F}-\left.\sigma^{i}\left(t_{I}\right) Q_{i}^{I}\right|_{*},
$$

for solutions which interpolate between boundary conditions imposed on hypersurfaces located at Euclidean times $t_{F}$ and $t_{I}$. If we compare this to the instanton action (14) found when computing the instanton amplitude using the two axionic actions,

$$
\Sigma_{*}=\beta^{i}\left(t_{F}\right) Q_{i}^{F}-\left.\beta^{i}\left(t_{I}\right) Q_{i}^{I}\right|_{*},
$$

we find that the results almost but not quite agree. The reason is that the relation $\partial_{m} \sigma^{i}= \pm \partial_{m} \beta^{i}$ only fixes the $\beta^{i}$ up to integration constants. As far as classical physics is concerned these integration constants are irrelevant because of the continuous shift symmetry. However, as we have seen, this symmetry is broken in the quantum theory to a discrete subgroup, and the quantum theory is sensitive to the values of the axions and hence to the integration constants $C^{i}=\sigma^{i}\left(t_{F}\right)-\beta^{i}\left(t_{F}\right)=\sigma^{i}\left(t_{I}\right)-\beta^{i}\left(t_{I}\right)$. Instanton actions based on axions and on antisymmetric tensor fields differ in general by the amount

$$
S_{\mathrm{inst}}-\Sigma_{*}=C^{i}\left(Q_{i}^{F}-Q_{i}^{I}\right) .
$$

If we insist that the quantum theories based on axions and antisymmetric tensor fields are equivalent, then we must impose that the $C^{i}$ vanish modulo the remaining discrete shift symmetries

$$
C^{i}=2 \pi i k, \quad k \in \mathbb{Z} .
$$

This is a restriction on the zero modes of the axion fields $b^{i}$, which classically are completely trivial. The subtlety that we find in the quantum equivalence between axions and antisymmetric tensor fields reflects that the axionic shift symmetry is a global symmetry which is broken generically by quantum effects, while the tensor field theory has a local gauge symmetry, which cannot. The condition (20) imposes that the charge sectors and saddle points of both theories match. We will see later that the relation (20) is equivalent to imposing that the instanton action equals the mass of the soliton obtained by dimensional lifting. 
Let us next discuss the relation between the Euclidean scalar-tensor action (17) and the two Euclidean scalar actions (2) and (3) in more detail. At the classical level the dualization is performed by first adding the Bianchi identies for the field strength $H_{m n p \mid i}$ with Lagrange multipliers, denoted $b^{i}$, and then eliminating the tensor fields by their equation of motion. Since details were given in [26], we only cite the final result

$$
\begin{aligned}
S_{E}[\sigma, b]= & \int d^{4} x\left(\frac{1}{2} N_{i j}(\sigma) \partial_{m} \sigma^{i} \partial^{m} \sigma^{j}-\frac{1}{2}(3 ! \lambda)^{2} N_{i j}(\sigma) \partial_{m} b^{i} \partial^{m} b^{j}\right) \\
& +(3 ! \lambda)^{2} \oint d^{3} \mathbf{x} n^{m} b^{i} N_{i j}(\sigma) \partial_{m} b^{j} .
\end{aligned}
$$

Here $\lambda$ is normalization constant. We observe that either the definite scalaraxion action (2) or the indefinite scalar-axion action (3) is obtained, with the same boundary term as in the amplitude calculation, by setting $(3 ! \lambda)^{2}=-1$ and $(3 ! \lambda)^{2}=1$, respectively. The first choice, corresponding to imaginary $\lambda$ preserves the definiteness of the action, but maps the real instanton solution to an imaginary saddle point. The second choice, corresponding to real $\lambda$, preserves the reality of the saddle point, but yields an indefinite Euclidean action. This is a particular feature of Euclidean signature. In Minkowski signature dualization simultanously preserves the saddle points and the positivity of kinetic terms. The fact that in the Euclidean formulations of axions we either loose definiteness of real saddle points confirmis our previous remarks that it is unavoidable, and in fact natural, to consider complex field configurations.

We also observe that the boundary term obtained by dualization contains the undifferentiated axions $b^{i}$. Therefore the resulting instanton action agrees with the one (14) obtained in the amplitude calculation. As a consequence, dualization does not preserve the value of the instanton action, unless we impose the condition (20). The same conclusion is reached if the dualization is performed at the level of the functional integral instead of the classical action. This could be demonstrated by repeating the calculation of the transition amplitude between axionic states. We prefer to consider the dualization of the partition function instead, because this shows that the subtleties related to the axionic zero modes even occur when no boundary conditions (corresponding to initial and final states) are imposed on the functional integral.

Since the scalar fields $\sigma^{i}$ are spectators in the dualization, we only consider the part of the partition function which depends on the tensor fields. The starting point is a simplified partition function 11 where the Bianchi identity is implemented through a functional delta function, so that we can perform an unrestricted integral over the field strenght $H_{m n p \mid i}$ :

$$
Z=\int D H \exp \left(-\int d^{4} x \frac{1}{2 \cdot 3 !} N^{i j} H_{m n p \mid i} H_{j}^{m n p}\right) \delta\left(\epsilon^{m n p q} \partial_{m} H_{n p q \mid k}\right) .
$$

The first step is to convert the functional delta function into an additional 'Fourier' functional integral:

$$
=\int D H D b \exp \left(-\int d^{4} x\left(\frac{1}{2 \cdot 3 !} N^{i j} H_{m n p \mid i} H_{j}^{m n p}-i \mu b^{i} \epsilon^{m n p q} \partial_{m} H_{n p q \mid i}\right)\right) .
$$

\footnotetext{
${ }^{11}$ When working with the $B$-field itself, one would need to include gauge fixing terms, ghosts, and ghosts for ghosts, since this is a reducible gauge theory, as reviewed in [42 43]. But these complications do not appear to be relevant to our purpose.
} 
Here $\mu$ is a constant that we will use later to obtain the conventional normalization of the resulting dual action 12 Next we perform an integration by parts, so that the $H$-field only occurs algebraically, or within boundary terms:

$$
\begin{aligned}
Z= & \int D H D b \exp \left(\int d^{4} x\left(-\frac{1}{2 \cdot 3 !} N^{i j} H_{m n p \mid i} H_{j}^{m n p}+i \mu \partial_{m} b^{i} \epsilon^{m n p q} H_{n p q \mid i}\right)\right. \\
& \left.-\mu i \int d^{4} x \partial_{m}\left(\epsilon^{m n p q} b^{i} H_{n p q \mid i}\right)\right) .
\end{aligned}
$$

Defining

$$
\tilde{H}_{m n p \mid i}=H_{m n p \mid i}-3 ! \mu i N_{i j} \epsilon_{m n p q} \partial^{q} b^{j}
$$

we can complete the square and shift integration variables $H_{m n p \mid i} \rightarrow \tilde{H}_{m n p \mid i}$ to obtain

$$
\begin{aligned}
Z= & \int D \tilde{H} D b \exp \left(-\int d^{4} x N^{i j} \tilde{H}_{m n p \mid i} \tilde{H}_{j}^{m n p}-\int d^{4} x(3 ! \mu) N_{i j} \partial_{m} b^{i} \partial^{m} b^{j}\right. \\
& \left.-\mu i \oint n^{m} b^{i} \epsilon_{m n p q} \tilde{H}_{i}^{n p q}+(3 ! \mu)^{2} \oint n^{m} N_{i j} b^{i} \partial_{m} b^{j}\right)
\end{aligned}
$$

The integration over $\tilde{H}_{m n p \mid i}$ decouples from the integration over $b^{i}$, except for one of the boundary terms. This term vanishes for on shell field configurations where $H_{m n p \mid i}$ and $\partial_{q} b^{i}$ are Hodge dual, and we assume that it can be dropped consistently within the semiclassical approximation. Given this, the integration over $\tilde{H}_{m n p \mid i}$ decouples and gives a (formal, infinite) multiplicative constant that we can ignore. The remaining functional integral for the fields $b^{i}$ is

$$
Z=\int D b \exp \left(-\int d^{4} x(3 ! \mu) N_{i j} \partial_{m} b^{i} \partial^{m} b^{j}+(3 ! \mu)^{2} \oint n^{m} N_{i j} b^{i} \partial_{m} b^{j}\right)
$$

which is the partition function associated with the definite Euclidean action (2), together with the boundary term. The standard normalization is obtained by choosing $(3 ! \mu)^{2}=1$, which implies that $\mu$ and the previous normalization constant $\lambda$ are related by $\mu= \pm i \lambda$. Within the functional framework it is natural to regard $\mu$ as a real constant, because the $b^{i}$ are introduced through the Fourier representation of the functional delta function implementing the Bianchi identity. Then the definiteness of the Euclidean action is preserved, so that the functional integral remains damped. However, we cannot avoid completely to consider complex field values: the shift in the integration over the $H$-fields is imaginary, and the real saddle points of the tensor action which correspond to instantons become imaginary saddle points upon dualization. Thus we need to consider complex values of the axion fields $b^{i}$ to match the semiclassical expansions of both version of the theory. Moreover, the presence of the boundary term breaks the continuous shift symmetry of the bulk action once we consider field configurations which have support on these boundary terms. This feature has no counter part in the tensor field partition function.

\footnotetext{
${ }^{12}$ We will find that $\mu$ is related to the normalization constant occuring in the 'classical' dualization by $\mu= \pm i \lambda$.
} 


\section{$5 \quad$ Relating instantons to solitons}

To round up our investigations, we now briefly relate them to the results of [26], where the same class of instanton solutions was used to generate solitons and black holes by dimensional lifting. The main point is that instanton action is directly related to the mass of the solutions obtained by lifting.

\subsection{Lifting without gravity}

As shown in [26] the indefinite Euclidean action (3) can be lifted with respect to time to a theory of real scalars and abelian gauge fields of the form

$$
S=\int d^{5} x\left(-\frac{1}{2} N_{i j}(\sigma) \partial_{\mu} \sigma^{i} \partial^{\mu} \sigma^{J}-\frac{1}{4} F_{\mu \nu}^{i} F^{j \mid \mu \nu}\right),
$$

where $\mu, \nu=0,1,2,3,4$ are five-dimensional Lorentz indices. The four-dimensional scalars $b^{i}$ are related to the five-dimensional gauge fields $A_{\mu}^{i}$ by $b^{i}=-A_{0}^{i}$. The scalar action (3) does not account for the magnetic components $F_{m n}^{i}$ of the fivedimensional gauge fields. However, (3) is a consistent truncation in the sense that any solution of (3) lifts to a static, purely electric solution of (23). If one uses (3) to generate five-dimensional solitons, one could add further terms to the five-dimensional action as long as they do not contribute to static, purely electric backgrounds. One example, which occurs in supersymmetric theories, is a Chern-Simons term. The lifting works without imposing any restrictions on the scalar metric $N_{i j}$, bu to obtain explicit solutions in terms of harmonic functions we impose that $N_{i j}$ is a Hessian metric. Upon reduction over time we obtain para-Kähler metric on the resulting extended scalar manifold $\mathcal{E}$. Let us also note that by dimensional reduction over space we obtain the Minkowski signature action (1) with its positive definite scalar manifold $\mathcal{M}$. If the actions (23), (3) and (10) are parts of supersymmetric actions, then $N_{i j}$ is subject to additional constraints.

In [20, 26] it was shown that instanton solutions of (3), where the harmonic functions are taken to be of the single centered type,

$$
H_{i}(r)=A_{i}+\frac{B_{i}}{r^{2}}
$$

lift to solitonic solutions with electrical charge $Q_{i} \propto B_{i}$ and mass

$$
M=\sigma^{i}(\infty) Q_{i} .
$$

Here the mass is defined as the integral of the energy density, given by the component $T_{00}$ of the energy momentum tensor, over space. In comparison to the amplitude calculation, the role of the boundary is played by an asymptotic three-sphere at infinity, and not by hyperplanes at infinite Euclidean time. The center $r=0$ of the harmonic function also needs to be treated as a boundary. Proper solitons correspond to solutions where the centers do not contribute, and it was shown in [26] that this is the case if the Hesse potential is a homogeneous function of non-positive degree. For Hesse potentials of positive degree the contribution from the center is infinite, so that such theories do not admit solitonic solutions of this type 13 In the language of $p$-branes, the center $r=0$

\footnotetext{
${ }^{13}$ As we will see below, this is equivalent to the statement that the reduced Euclidean theory does not have instantons, because the saddle point solution has infinite action.
} 
is the location of a 0-brane of the five-dimensional theory, which becomes a $(-1)$ brane upon reduction over time. The tensions of $p$-branes are related by dimensional reduction, and for 0 -branes and $(-1)$ branes the mass and action respectively play the role of the tension. Thus we expect that the mass of the soliton equals the action of the instanton from which it was generated 14 Since the instanton action is

$$
S_{\mathrm{inst}}=b^{i}(\infty) Q_{i}
$$

we find that $M=S_{\text {inst }}$ requires $b^{i}(\infty)=\sigma^{i}(\infty)$, or $C^{i}=0$, which is the same condition as we found when describing instanton solutions in terms of the dual tensor field theory. Note that like dualization dimensional lifting has the effect to convert the axionic shift symmetries into proper gauge symmetries. The soliton mass cannot depend on $b^{i}$ since this would make it gauge dependent.

For completeness let us mention that there are more general solutions based on multi-centered harmonic functions

$$
H_{i}(\mathbf{x})=A_{i}+\sum_{a=1}^{N} \frac{B_{i, a}}{\left|\mathbf{x}-\mathbf{x}_{a}\right|^{2}}
$$

which describe static configurations of solitons. For such solutions the total energy is the sum of the masses of the solitions, thus reflecting the saturation of a Bogomol'nyi bound. The relation between this Bogomol'nyi bound and the one for instantons will be explained later for the special case of supersymmetric (BPS) solutions.

\subsection{Lifting with gravity}

Solutions of the indefinite Euclidean action (3) which satisfy the extremal instanton ansatz $\partial_{m} \sigma^{i}= \pm \partial_{m} \beta^{i}$ remain solutions, without modification, if we add a four-dimensional Euclidean Einstein-Hilbert term

$$
S=\frac{1}{2} \int d^{4} x \sqrt{g_{(4)}}\left(-R_{(4)}+N_{i j} \partial_{m} \sigma^{i} \partial^{m} \sigma^{j}-N_{i j} \partial_{m} b^{i} \partial^{m} b^{j}\right) .
$$

The resason is that (44) implies that the energy momentum tensor vanishes, so that the four-dimensional Euclidean Einstein equations are solved by a flat metric $g_{m n}=\delta_{m n}$ (or more generally by a Ricci-flat metric). The resulting solutions of (24) can be lifted to extremal black hole solution of a five-dimensional action of the following form [26]:

$$
S=\int \sqrt{g_{(5)}} d^{5} x\left(\frac{1}{2} R_{(5)}-\frac{3}{4} a_{i j}(h) \partial_{\mu} h^{i} \partial^{\mu} h^{j}-\frac{1}{4} a_{i j}(h) F_{\mu \nu}^{i} F^{j \mid \mu \nu}\right) .
$$

The presence of gravity complicates the dimensional lifting/reduction considerably. In the following we give a concise summary, and refer to [20, 26] for more details. The space-time metrics are related by

$$
d s_{(5)}^{2}=-e^{2 \tilde{\sigma}}\left(d t+A_{m} d x^{m}\right)^{2}+e^{-\tilde{\sigma}} d s_{(4)}^{2},
$$

\footnotetext{
${ }^{14}$ There is a numerical constant proportional to the volume of the compactified direction which we take to be 1 here. Our normalization of the five-dimensional electric charge is such that it is equal to the axionic charge.
} 
where $\tilde{\sigma}$ is the Kaluza Klein scalar and $A_{m}$ is the Kaluza-Klein vector. When lifting instanton solutions, then $A_{m}=0$ and $d s_{(4)}^{2}=\delta_{m n} d x^{m} d x^{n}$, so that we obtain the line element of an extremal static black hole solution (which can be single or multi-centered):

$$
d s_{(5)}^{2}=-e^{2 \tilde{\sigma}} d t^{2}+e^{-\tilde{\sigma}} \delta_{m n} d x^{m} d x^{n} .
$$

The only non-trivial component of the five-dimensional metric is the KaluzaKlein scalar, which is obtained by solving the four-dimensional scalar equations of motion. The Kaluza-Klein vector can be truncated out consistently, because we only consider non-rotating solutions. Since the metric contributes one fourdimensional scalar, we need to start with $n-1$ real scalars and $n$ gauge fields in five dimensions to obtain a scalar action of the form (24) with $2 n$ real scalars. This can be implemented by adapting a construction well known form fivedimensional vector multiplets [36, 26. Instead of working with $n-1$ independent five-dimensional scalars, one uses $n$ five-dimensional scalars $h^{i}$ which are subject to the constraint

$$
\hat{\mathcal{V}}(h)=1
$$

The constraint eliminates one independent degree of freedom. In supergravity the function $\mathcal{V}(h)$ is called the prepotential, and must be a homongeneous cubic polynomial. If we do not require supersymmetry we can relax this condition and admit any prepotential which is a homogeneous function of arbitrary degree $p$. As in supergravity we require that the scalar metric $a_{I J}(h)$ is Hessian, with Hesse potential

$$
\mathcal{V}(h)=-\frac{1}{p} \log \hat{\mathcal{V}}(h) .
$$

Such a Hesse potential is almost, but not quite, homogeneous of degree zero, and its $k$-th derivatives are homogeneous functions of degee $-k$. In particular, the scalar metric $a_{i j}$ is homogeneous of degree -2 . This feature is crucial in showing that upon dimensional reduction (25) becomes (24), plus terms which are not relevant for static, purely electric solutions. The key step is to combine the constrained five-dimensional scalars $h^{i}$ with the Kaluza-Klein scalar $\tilde{\sigma}$ into $n$ unconstrained real four-dimensional scalars

$$
\sigma^{i}=e^{\tilde{\sigma}} h^{i}
$$

One can then use the homogeneity properties of $a_{i j}$ to obtain (24). The scalar metric $N_{i j}(\sigma)$ is proportional to $a_{i j}(h)$.

It has been shown in 26] that by using instanton solutions based on single and multi-centered harmonic functions one obtains single and multi-centered extremal black hole solution in five-dimensions, which share all essential features of BPS black holes of five dimensional vector multiplets. In particular, one obtains global, algebraic attractor equations, which express the solution in terms of harmonic functions, by rewriting (5) in terms of five-dimensional variables. Using the five-dimensional version of the ADM mass formula, one finds

$$
M_{A D M}=\frac{3}{2} \oint d^{3} \mathbf{x} n^{m} e^{-\tilde{\sigma}} \partial_{m} \tilde{\sigma},
$$


where the integral is over an asymptotic three-sphere 15 If one expresses the instanton action in terms of five-dimensional quantities, one obtains

$$
S_{\text {inst }}=\frac{3}{2} \oint d^{3} \mathbf{x} n^{m} \partial_{m} \tilde{\sigma} .
$$

While the integrands of the surface integrals for $M_{A D M}$ and $S_{\text {Inst }}$ are different, the factor $e^{-\tilde{\sigma}}$ does not contribute, due to its fall off behaviour for black hole solutions [26. Therefore ADM mass and instanton action agree, provided that we impose $\sigma^{i}(\infty)=b^{i}(\infty)$ as discussed before.

\section{Instantons solutions for Euclidean $N=2$ vec- tor multiplets}

We now turn to a particular class of examples, instanton solutions for fourdimensional Euclidean $N=2$ vector multiplets and the corresponding solitonic solutions for five-dimensional vector multiplets and black hole solutions for fivedimensional supergravity with vector multiplets. The full actions contain of course further terms besides those in (3), (23), (25), but for static purely electric backgrounds these additional terms are not relative. As far as solving the field equations is concerned, we are just dealing with a particular subclass of the models considered previously. Therefore we focus on the additional features due to supersymmetry. We show how the extremal instanton ansatz can be derived by imposing a Euclidean BPS condition, and verify explicitly that the resulting solutions are supersymmetric from both the four-dimensional and the five-dimensional point of view.

\subsection{The supersymmetry algebra}

We start with the minimal five-dimensional supersymmetry algebra, from which the four-dimensional Euclidean supersymmetry algebra can be obtained by dimensional reduction over time [20]. All fermions, including the supercharges and the supersymmetry transformation parameters are taken to be symplectic Majorana spinors. If $\lambda^{i}$, where $i=1,2$, is a pair of complex spinors, then the symplectic Majorana condition is

$$
\left(\lambda^{i}\right)^{*}=C \gamma_{0} \epsilon_{i j} \lambda^{j} .
$$

Here $C$ is the charge conjugation matrix, which satisfies

$$
C^{T}=-C, \quad \gamma^{\mu T}=C \gamma^{\mu} C^{-1} .
$$

For definiteness we take $C$ to be real, $C=C^{*}$. Here and in the following we usually supress spinor indices $\alpha, \beta=1, \ldots, 4$ :

$$
\lambda^{i}=\left(\lambda_{\alpha}^{i}\right), \quad \gamma^{\mu}=\left(\gamma_{\alpha}^{\mu \beta}\right), \quad C=\left(C^{\alpha \beta}\right), \quad C^{-1}=\left(C_{\alpha \beta}^{-1}\right) .
$$

The symplectic Majorana condition can be imposed in five-dimensional Minkowski space, four-dimensional Minkowski space and four-dimensional Euclidean space,

\footnotetext{
${ }^{15}$ As for solitons based on Hesse potentials which are homogeneous functions of non-positive degree, there are no contributions from the centers.
} 
because in all these cases the charge conjugation matrix can be chosen to satisfy (27) 20.

The minimal five-dimensional supersymmetry algebra takes the form

$$
\left\{Q_{i \alpha}, Q_{j \beta}\right\}=-\frac{1}{2} \epsilon_{i j}\left(\gamma^{\mu} C^{-1}\right)_{\alpha \beta} P_{\mu} .
$$

The indices $i=1,2$ are raised and lowered with $\epsilon_{i j}$ and its inverse 16 and $\mu, \nu=$ $0,1,2,3,5$ are five-dimensional Lorentz vector indices. The five-dimensional supersymmetry algebra has the R-symmetry group $S U(2)_{R}$, under which symplectic Majorana spinors transform as a doublet.

The four-dimensional Euclidean supersymmetry algebra is obtained by dimensional reduction over time. If we restrict ourselves to states on which $P_{0}$ operates trivially, we obtain

$$
\left\{Q_{i \alpha}, Q_{j \beta}\right\}=-\frac{1}{2} \epsilon_{i j}\left(\gamma^{m} C^{-1}\right)_{\alpha \beta} P_{m},
$$

where $m=1,2,3,5$ are the four Euclidean directions. The corresponding Clifford algebra is generated by $\gamma^{m}$. Observe that $\gamma^{0}$ now plays the role of a 'chirality operator', and assumes the role played by $\gamma_{5}$ in four-dimensional Minkowski space. The R-symmetry group is enhanced to $S O(1,1)_{R} \times S U(2)_{R}[20$. The non-compact abelian factor is generated by $\gamma_{0}$ and acts chirally:

$$
Q_{i} \rightarrow e^{-i \gamma^{0} \phi} Q_{i}
$$

where $\phi$ is real. Since $\gamma_{0}$ is anti-Hermitean, $-i \gamma_{0}$ has real eigenvalues \pm 1 , and the R-symmetry group $S O(1,1))_{R}$ acts by chiral scale transformations.

In four-dimensional Minkowski space $\gamma_{0}$ is replaced by $\gamma_{5}$, which is Hermitean. The resulting R-symmetries act by

$$
Q_{i} \rightarrow e^{i \gamma_{5} \phi} Q_{i}
$$

Since $i \gamma_{5}$ has eigenvalues $\pm i$, these are chiral phase transformations, and the resulting R-symmetry group is $U(1)_{R}$.

To discuss BPS states, we need to add central charges. The five-dimensional algebra admits are real central charge $R$ :

$$
\left\{Q_{i \alpha}, Q_{j \beta}\right\}=-\frac{1}{2} \epsilon_{i j}\left(\gamma^{\mu} C^{-1}\right)_{\alpha \beta} P_{\mu}+\frac{i}{2} \epsilon_{i j} C_{\alpha \beta}^{-1} R .
$$

The four-dimensional Euclidean supersymmetry algebra is again obtained by reduction over time, and this time we keep $P_{0}$ which becomes the second real central charge:

$$
\left\{Q_{i \alpha}, Q_{j \beta}\right\}=-\frac{1}{2} \epsilon_{i j}\left(\gamma^{m} C^{-1}\right)_{\alpha \beta} P_{m}-\frac{1}{2} \epsilon_{i j}\left(\gamma^{0} C^{-1}\right)_{\alpha \beta} P_{0}+\frac{i}{2} \epsilon_{i j} C_{\alpha \beta}^{-1} R .
$$

Similarly, when reducing with respect to the extra space direction $\mu=5$, the operator $P_{5}$ becomes a central charge, which is usually combined with $R$ into a complex central charge.

\footnotetext{
${ }^{16}$ We use the NW-SE convention, see 20] for more details on our conventions.
} 


\subsection{BPS states}

A systematic way to obtain the BPS states related to a supersymmetry algebra is to require that the supersymmetry transformation parameters form a zero eigenvector of the Bogomol'nyi matrix 17 , which is the matrix formed by the anticommutators of supercharges,

$$
\left\{Q_{i \alpha}, Q_{j \beta} \epsilon^{j \beta}=0 .\right.
$$

This eigenvalue problem is the integrability condition resulting from imposing that the BPS state is invariant under the supersymmetry transformation generated by $\epsilon^{j \beta} Q_{j \beta}$, and the eigenvectors $\epsilon^{j \beta}$ are the transformation parameters of the supersymmetry transformation which leaves the BPS state invariant.

Let us first consider the five-dimensional case where this relation becomes

$$
\left(-\frac{1}{2} \epsilon_{i j}\left(\gamma^{\mu} C^{-1}\right)_{\alpha \beta} P_{\mu}+\frac{i}{2} \epsilon_{i j} C_{\alpha \beta}^{-1} R\right) \epsilon^{j \beta}=0
$$

upon using the algebra. If we impose that the BPS state is massive, we can go to its rest frame where $P_{m}=0$. Then $P_{0}=M$ is the mass and we have:

$$
\left(-\frac{1}{2} \epsilon_{i j}\left(\gamma^{0} C^{-1}\right)_{\alpha \beta} M+\frac{i}{2} \epsilon_{i j} C_{\alpha \beta}^{-1} R\right) \epsilon^{j \beta}=0 .
$$

We use $\epsilon_{i j}$ to lower the $S U(2)_{R}$ index, apply $C$ to the equation and use that $C \gamma^{0} C^{-1}=\gamma^{0 T}$ :

$$
-M \gamma_{\beta}^{0}{ }^{\alpha} \epsilon_{i}^{\beta}+i R \epsilon_{i}^{\alpha}=0 .
$$

BPS states saturate the Bogomol'nyi bound $M \geq|R|$, therefore $M= \pm R$. Since $\gamma_{0}$ can always be chosen to be either symmetric or antisymmetric, we obtain

$$
i \gamma^{0 \alpha}{ }_{\beta} \epsilon_{i}^{\beta}= \pm \epsilon_{i}^{\alpha} .
$$

Thus the 'Killing spinor' corresponding to a BPS state must be an eigenstate of $i \gamma^{0}$. Since the eigenvalues \pm 1 of $i \gamma^{0}$ are two-fold degenerate, we obtain four (real) Killing spinors and a state satisfying $M= \pm R$ is invariant under one half of the supersymmetry algebra.

This condition still makes sense in the Euclidean supersymmetry algebra, which is obtained by dimensional reduction over time. Only the interpretation changes: the five-dimensional mass $P_{0}=M$ is a central charge from the four-dimensional Euclidean point of view, and therefore $M=|R|$ is a relation between the two central charges of the algebra. In supergravity the central charge is related to the electric charge of the soliton. Upon dimensional reduction, this becomes the 'instanton charge', which in the models we will consider is the axionic charge. The central charge $M$ should also have a physical interpretation, the natural candidate being the instanton action. Then the BPS relation $M=|R|$ becomes a relation between instanton action and instanton charge from the four-dimensional Euclidean point of view. Since $\gamma_{0}$ plays the role of the chirality operator in the Euclidean Clifford algebra, the condition imposed on the supersymmetry parameters is a chirality condition. As we will see below, this implies that bosonic BPS field configuration satisfy (4), which should be viewed as a self-duality condition.

\footnotetext{
${ }^{17}$ We refer to 39 for a review of this method.
} 


\subsection{The Euclidean $\mathcal{N}=2$ Vector Multiplet}

Off shell four-dimensional Euclidean vector multiplets were constructed in [20], to which we refer for details. In the following we use the same conventions as in 20] and label vector multiplets, and, hence, scalars by captial indices $I, J, \ldots=1, \ldots, n$. In previous sections these indices were denoted $i, j, \ldots$, which in this section we reserve for the $S U(2)_{R}$ indices.

The field content of a Euclidean off-shell vector multiplet is as follows:

$$
\left(X^{I}, \lambda^{I i}, A_{m}^{I} \mid Y^{i j I}\right)
$$

Here $X^{I}$ are scalars, $\lambda^{I i}$ fermions, $A_{m}^{I}$ gauge fields, and $Y^{i j I}$ are auxiliary fields. While the scalars are coordinates on the scalar manifold $\mathcal{E}$, the other fields carrying a manifold index are sections of its tangent bundle $T \mathcal{E}$.

As we saw above, when going from Minkowski to Euclidean signature the $R$-symmetry group of the $N=2$ supersymmetry changes from $S U(2)_{R} \times U(1)_{R}$ to $S U(2)_{R} \times S O(1,1)_{R}$. This was already observed in [18] who also observed that this changes the metric of the scalar manifold for vector multiplets. In [20] this was explored systematically. The generator of the abelian factor of the $R$ symmetry group acts isometrically on the scalar manifold. While a $U(1)_{R}$ factor implies that the scalar manifold carries a complex structure, its non-compact form $S O(1,1)_{R}$ implies that the complex structure is replaced by a para-complex structure. In the following we elaborate on the brief discussion of para-complex geometry given in Section 3, and refer to [20, 17. for a comprehensive account.

Concerning the scalar fields $X^{I}$, which are interpreted as coordinates on $\mathcal{E}$, there are two possible descriptions. One option, which we already used in Section 3 is to take them to be para-complex coordinates

$$
X^{I}=\sigma^{I}+e b^{I},
$$

which stresses the analogy with the complex fields $X^{I}=\sigma^{I}+i b^{I}$ used in the Minkowski version of the theory. This is natural, but there is alternative choice, which avoids using the para-complex unit $e$, namely 'null' or 'lightcone' coordinates

$$
X_{ \pm}^{I}=\sigma^{I} \pm b^{I} .
$$

This alternative description is not available in Minkowski signature, because a complex structure has imaginary eigenvalues $\pm i$. Therefore its eigenvectors cannot be real, and one is forced to work with the complexified tangent bundle. In constrast, a para-complex structure has real eigenvales \pm 1 and real eigenvectors. The null coordinates go along the integral curves of these eigenvectors and thus provide adapted coordinates. Therefore one can work with adapted real coordinates and the real tangent bundle $T \mathcal{E}$, thus avoiding to make use of the para-complex unit $e$. However, we prefer to use para-complex coordinates, and to use the para-complexified tangent bundle $T \mathcal{E}_{C}$ because of the close analogy with the complex case. In particular, formulae written in local coordinates can be translated systematically from Minkowski to Euclidean space by substituting $i \rightarrow e$, for all factors of $i$ related to the complex structure of the scalar target space. A more geometric way of expressing this is that we replace the complex scalar manifold $\mathcal{M}$ and its complexified tangent bundle $T \mathcal{M}_{\mathbb{C}}$ by the para-complex manifold $\mathcal{E}$ and its para-complexified tangent bundle $T \mathcal{E}_{C}$. 
Besides the scalars $X^{I}$, the vector multiplets contain spinors $\lambda^{i I}$, vector fields $A_{m}^{I}$ and auxiliary fields $Y_{i j}^{I}$. All these fields carry a manifold index $I$ and can therefore be interpreted as sections of the tangent bundle of $\mathcal{E}$, either the real tangent bundle $T \mathcal{E}$ or its para-complexified version $T \mathcal{E}_{C}$. Since we prefer the para-complex version, we need to comment on some of its particular features.

The spinors $\lambda^{i I}$ are sections of the product bundle $T \mathcal{E}_{C} \times S$, where $S$ is the spin bundle. Since spinors are complex, $S$ carries a complex structure $\tilde{I}$. When going from Minkowski to Euclidean signature the underlying Clifford algebra changes signature, but $S$ is complex in both cases 18 On the product bundle the para-complex structure $J$ of $\mathcal{E}$ acts as $J \otimes \mathbb{1}$, while $\tilde{I}$ acts as $\mathbb{1} \otimes \tilde{I}$. It is manifest that both structures commute:

$$
(\mathbb{1} \otimes \tilde{I})(J \otimes \mathbb{1})=J \otimes \tilde{I}=(J \otimes \mathbb{1})(\mathbb{1} \otimes \tilde{I}),
$$

because they operate on different factors of the product $T \mathcal{E}_{C} \times S$. In terms of local coordinates, this amounts to $i e=e i$.

When working in local coordinates it is not always obvious how to translate formulae of the Minkowski theory to formulae in the Euclidean theory, because in the Minkowski theory there are two types of ' $i$ ', those related to the complex structure of the target space, and those related to the spinor representation. Only the first ones are replaced by $e$ 's, and any ambiguity has to be resolved by identifying which complex structure is behind a given factor of $i$ in the Minkowski theory. 19

After this general discussion, we list some formulae that we will need later on. Within the para-complex formalism, the spinors $\lambda^{i I}$ can be decomposed into two para-complex 'chiral' parts

$$
\lambda^{i I}=\lambda_{+}^{i I}+\lambda_{-}^{i I}
$$

where

$$
\lambda_{ \pm}^{i I}=\Gamma_{ \pm} \lambda^{i I}, \quad \Gamma_{ \pm}=\frac{1}{2}\left(\mathbb{1} \pm e(-i) \gamma^{0}\right) .
$$

To understand the geometrical, coordinate independent meaning of these formulae, first note that the symplectic Majorana condition is a reality condition on $S$, not on $T \mathcal{E}_{C}$. Thus it involves the $i$ 's but not the $e$ 's. Second, we take the fermions to be sections of the para-complexified tangent bundle, which can be decomposed into a para-holomorphic subbundle with eigenvalue $+e$ and an anti-para-holomorphic subbundle with eigenvalue $-e$, with respect to the paracomplex structure. The above projection acts on both factors of the product $T \mathcal{E}_{C} \times S$ simultanously and has para-complex eigenvalues $\pm e$. We will see below that this is convenient, because when using the para-complex spinors $\lambda_{ \pm}^{i}$ the supersymmetry transformations take exactly the same form in Minkowski and Euclidean signature.

When using adapted coordinates (39), there are no $e$ 's in the formulae but there is an analogous chiral decomposition

$$
\lambda^{i I}=\xi_{+}^{i I}+\xi_{-}^{i I}
$$

\footnotetext{
18 To avoid confusion, we emphasize that the complex structure of $S$ is not replaced by a para-complex structure when going to Euclidean signature.

${ }^{19}$ When working with complexified axions, there is yet another complex structure, which needs to be distinguished from the two discussed here. See the appendix of [26] for a formalism which helps to disentangle these complex structures when working in local coordinates.
} 
where

$$
\xi_{ \pm}^{i I}=\Gamma_{ \pm} \xi^{i I}, \quad \Gamma_{ \pm}=\frac{1}{2}\left(\mathbb{1} \pm(-i) \gamma^{0}\right) .
$$

The projector is now real valued, and looks similar to the normal four-dimensional chiral projection, with $\gamma_{5}$ replaced by $\gamma^{0}$.

Each vector multiplet also contains a gauge field $A_{m}^{I}$, with corresponding field strength $F_{m n}^{I}=2 \partial_{[m} A_{n]}^{I}$. When working in para-complex coordinates, we define the self-dual and anti-self-dual parts of the fields strengths as elements of the para-complex tangent space:

$$
F_{ \pm \mid m n}^{I}=\frac{1}{2}\left(F_{m n}^{I} \pm \frac{1}{e} \tilde{F}_{m n}^{I}\right) .
$$

As for the fermions, the reason for introducing para-complex fields is that the supersymmetry transformations take the same form as in Minkowski signature. When using adapted coordinates, one would instead take the standard Euclidean decomposition

$$
F_{ \pm \mid m n}^{I}=\frac{1}{2}\left(F_{m n}^{I} \pm \tilde{F}_{m n}^{I}\right)
$$

Finally, the balance of the off-shell degrees of freedom is provided by three real scalar fields, which form a triplet under $S U(2)_{R}$. They are organised into a symmetric tensor,

$$
Y^{i j I}=Y^{j i I}
$$

which is subject to the reality condition

$$
\left(Y^{i j I}\right)^{*}=Y_{i j}^{I}=\epsilon_{i k} \epsilon_{j l} Y^{k l I} .
$$

In the para-complex formalism, the supersymmetry transformations take the following form [20]:

$$
\begin{aligned}
\delta X^{I} & =i \bar{\epsilon}_{+}^{i} \lambda_{+}^{I} \\
\delta \bar{X}^{I} & =i \bar{\epsilon}_{-} \lambda_{-}^{I} \\
\delta \lambda_{+}^{i I} & =-\frac{1}{4} \gamma^{m n} F_{-m n}^{I} \epsilon_{+}^{i}-\frac{i}{2} \not \partial X^{I} \epsilon_{-}^{i}-Y^{i j I} \epsilon_{+j} \\
\delta \lambda_{-}^{i I} & =-\frac{1}{4} \gamma^{m n} F_{+m n}^{I} \epsilon_{-}^{i}-\frac{i}{2} \not \partial \bar{X}^{I} \epsilon_{+}^{i}-Y^{i j I} \epsilon_{-j} \\
\delta A_{m}^{I} & =\frac{1}{2}\left(\bar{\epsilon}_{+} \gamma_{m} \lambda_{-}^{I}+\bar{\epsilon}_{-} \gamma_{m} \lambda_{+}^{I}\right) \\
\delta Y^{i j I} & =-\frac{1}{2}\left(\bar{\epsilon}_{+}^{(i} \not \partial \lambda_{-}^{j) I}+\bar{\epsilon}_{-}^{(i} \not \partial \lambda_{+}^{j) I}\right) .
\end{aligned}
$$

The supersymmetry transformations of the Minkowski signature theory take precisely the same form, with para-complex quantities replaced by complex quantities as explained before 20 Supersymmetry acts chirally in the sense that para-holomorphic scalars transform into positive chirality spinors which transform into anti-selfdual field strength.

The vector multiplet Lagrangian is gauge invariant, and therefore it does not involve the gauge field $A_{m}^{I}$ directly, but only the gauge invariant field strength

\footnotetext{
${ }^{20}$ When comparing to the literature one needs to take into account that most of the $N=2$ literature uses Majorana spinors, which do not exist in Euclidean signature. Instead we use symplectic Majorana spinors, which exist in both signatures. See 20] for the details.
} 
$F_{m n}^{I}$. The anti-self-dual part of the field strength is part of a restricted chiral multiplet

$$
\left(X^{I}, \lambda_{+}^{I i}, F_{-\mid m n}^{I} \mid Y^{i j I}\right),
$$

while the self-dual part belongs to the complex (and para-complex) conjugated multiplet. Since supersymmetry acts chirally it does not mix the above restricted chiral multiplet with its complex conjugate.

\subsection{Supersymmetric field configurations}

Our goal is to identify the BPS field configurations of Euclidean vector multiplets where the only excited fields are scalars. Therefore we set the fermions and the gauge fields to zero in (42), and obtain the following condition on the scalars $X^{I}$ and the auxiliary fields $Y^{i j I}$ :

$$
\begin{aligned}
\delta \lambda_{+}^{i I} & =-\frac{i}{2} \not \partial X^{I} \epsilon_{-}^{i}-Y^{i j I} \epsilon_{+j}=0 \\
\delta \lambda_{-}^{i I} & =-\frac{i}{2} \not \partial \bar{X}^{I} \epsilon_{+}^{i}-Y^{i j I} \epsilon_{-j}=0 \\
\delta Y^{i j I} & =0 .
\end{aligned}
$$

It is consistent to set $Y^{i j I}=0$, which is in fact the equation of motion of the auxiliary fields in a bosonic background. The remaining condition on the scalar is

$$
-\frac{i}{2} \not \partial X^{I} \epsilon_{-}^{i}=0
$$

together with its complex conjugate 21 Like the fermions $\lambda^{i I}$, the supersymmetry transformations parameter $\epsilon^{i}$ are symplectic Majorana spinors, and they have been decomposed into para-complex chiral components

$$
\begin{gathered}
\epsilon^{i}=\epsilon_{+}^{i}+\epsilon_{-}^{i}, \\
\epsilon_{ \pm}^{i}=\frac{1}{2}\left(\epsilon^{i} \pm e(-i) \gamma_{0} \epsilon^{i}\right) .
\end{gathered}
$$

From our analysis of the algebra we have seen that the BPS condition for a point-like object (an instanton in four dimensions, a soliton in five dimensions) is either

$$
\gamma_{0} \epsilon^{i}=-i \epsilon^{i}
$$

or

$$
\gamma_{0} \epsilon^{i}=i \epsilon^{i}
$$

Each of these choices reduces the number of independent supersymmetry parameters from 8 to 4 . Therefore invariant field configurations have 4 Killing spinors and are ' $\frac{1}{2}$-BPS'. To work out the resulting condition on the scalars $X^{I}$, let us make the choice (50) for definiteness. Then

$$
\epsilon_{\mp}^{i}=\frac{1}{2}(1 \mp e) \epsilon^{i},
$$

\footnotetext{
${ }^{21}$ Here and in the following 'complex conjugate' as a short hand for taking the para-complex conjugation of scalars and tangent vectors, and the complex conjugate of spinors.
} 
and the constraint on scalar fields becomes

$$
\not \partial\left(\sigma^{I} \pm e b^{I}\right)(1 \mp e) \epsilon^{i}=0 .
$$

We want to find solutions which do not require to set the scalars to constant values, which would lead to a fully supersymmetric ground state solution. While this would be impossible in Minkowski signature with its complex target space, in Euclidean signature with its para-complex geometry we can make use of the identity

$$
(1+e)(1-e)=0 .
$$

This provides us with a non-trivial solution,

$$
\not \partial \sigma^{I}=\not \partial b^{I} .
$$

If we choose to impose the other possible constraint (51) on the supersymmetry parameters, we obtain $\not \partial \sigma^{I}=-\not \partial b^{I}$ Combining both cases we see that the condition for a scalar field configuration to be $\frac{1}{2}$-BPS is:

$$
\not \partial \sigma^{I}= \pm \not \partial b^{I} .
$$

Since the $\gamma$-matrices are linearly independent, this is equivalent to

$$
\partial_{m} \sigma^{I}= \pm \partial_{m} b^{I}
$$

which is the extremal instanton ansatz (4) 22

The geometrical interpretation of this condition is that the solution can only vary along the eigendirections of the para-complex structure. Looking back at the construction of solutions for general class of actions we see that we still obtain solutions if we relax the condition that the relative sign must be the same for all values of $I$. However, such solutions, while extremal, are not BPS. Thus by flipping signs we can generate non-BPS extremal solutions from BPS solutions. This observation agrees with the general pattern observed in the context of black holes. The target space metric of our model

$$
d s_{\mathcal{E}}^{2}=N_{I J}(\sigma)\left(d \sigma^{I} d \sigma^{J}-d b^{I} d b^{J}\right)
$$

has manifest discrete isometries $R_{J}^{I}$ acting by

$$
b^{I} \rightarrow R_{J}^{I} b^{J}= \pm b^{I}
$$

satisfying

$$
N_{I J} R_{k}^{I} R_{L}^{J}=N_{K L} .
$$

Such isometries generate new extremal solutions which are related to the old ones by rotating the axion charges, as was realized for black holes in [44, 45]. The BPS condition allows to reduce the field equations to gradient flow equations driven by the central charge. This feature carries over to extremal non-BPS solutions with the flow now being driven by a 'fake superpotential'. For instanton solutions this was discussed in some detail in [26].

${ }^{22}$ Remember that the fields $b^{I}$ were denoted $\beta^{i}$ in the previous sections. 


\subsection{BPS condition in adapted coordinates}

For completeness, let us show explicitly how the same conclusions can be arrived at when using the adapted real coordinates $X_{ \pm}^{I}=\sigma^{I} \pm b$ instead ot the paracomplex coordintes $X^{I}=\sigma^{I}+e b^{I}$. Then the Euclidean BPS condition for a purely scalar background is [20]:

$$
\begin{aligned}
\delta \xi_{+}^{i I} & =-\frac{i}{2} \not \partial X_{+}^{I} \eta_{-}^{i}, \\
\delta \xi_{-}^{i I} & =-\frac{i}{2} \not \partial X_{-}^{I} \eta_{+}^{i},
\end{aligned}
$$

where $\xi_{ \pm}^{i I}=\frac{1}{2}\left(\mathbb{1} \pm(-i) \gamma^{0}\right) \xi^{i I}$ are the chiral projections (with respect to $\gamma^{0}$ ) of the vector multiplet fermions and $\eta_{ \pm}^{i}=\frac{1}{2}\left(\mathbb{1} \pm(-i) \gamma^{0}\right) \epsilon^{i}$ are the chiral projections of the supersymmetry parameters.

Imposing $i \gamma^{0} \epsilon^{i}=\epsilon^{i}$ implies $\eta_{+}^{i}=0$ and the condition on the scalar fields is

$$
\not \partial X_{+}^{I}=0 \Leftrightarrow \partial_{m} \sigma^{I}=-\partial_{m} b^{I} .
$$

If we impose instead $i \gamma^{0} \epsilon^{i}=-\epsilon^{i}$, then $\eta_{+}^{i}=0$ and the condition on the scalars is

$$
\not \partial X_{-}^{I}=0 \Leftrightarrow \partial_{m} \sigma^{I}=\partial_{m} b^{I} .
$$

Combining both cases we recover (54).

\subsection{Extension to supergravity}

So far our discussion was based on rigid Euclidean vector multiplets. As discussed in Section 5 we can also couple the sigma model to gravity and lift instantons to black hole solutions. We would therefore like to check that the Euclidean BPS solutions obtained in this section lift to BPS black holes. One way of doing this would be to work out the Euclidean supersymmetry transformations for Euclidean vector multiplet coupled to supergravity. This has not been done yet, but at least the bosonic part of the action was worked out in [17. We can still use the general fact that extremal instanton solutions can be constructed consistently taking the Euclidean metric to be flat, because the extremality condition implies that the energy momentum tensor vanishes. Therefore we can consistently truncate the bosonic action found in [17] to its scalar sector. Then the only difference between the rigid and the local model is the condition imposed on the scalar metric $N_{I J}$. In the rigid case $N_{I J}$ must be an affine special para-Kähler metric [20. In terms of local coordinates this means that $N_{I J}$ has a para-Kähler potential $K(X, \bar{X})$

$$
N_{I J}=\frac{\partial^{2} K(X, \bar{X})}{\partial X^{I} \partial \bar{X}^{J}}
$$

which can be obtained from para-holomorphic prepotential $F(X)$ by

$$
K(X, \bar{X})=-e\left(X^{I} \bar{F}_{I}-F_{I} \bar{X}^{I}\right) .
$$

For models obtained by dimensional reduction of five-dimensional vector multiplets over time, the prepotential is homogeneous cubic polynomial $F(X)=$ $C_{I J K} X^{I} X^{J} X^{K}$. 
In the local case the para-Kähler form instead takes the form

$$
K(X, \bar{X})=-\log \left[-e\left(X^{M} \bar{F}_{M}-F_{M} \bar{X}^{M}\right)\right]
$$

where $M, N=0,1, \ldots, n$. The prepotential depends on one additional variable $X^{0}$, but is required to be homogeneous of degree two, so that the scalar metric only depends on $n$ of the $n+1$ variables. For models obtained by dimensional reduction of five-dimensional vector multiplets, the prepotential takes the form $F(X)=\left(X^{0}\right)^{-1} C_{I J K} X^{I} X^{J} X^{K}$.

Since the off-shell supersymmetry transformation rules given for rigid Euclidean vector multiplets do not depend on the prepotential, it is clear that we will have the same transformations in supergravity if we impose a flat metric. Therefore the condition for scalar $\frac{1}{2}$-BPS is not modified. In fact, this type of reasoning can be applied generally to asymptotically flat solutions of supergravity to find the algebraic structure of Killing spinors by looking for eigenvectors of the matrix of supersymmetry anticommutators, see for example [39. We can check this by comparing our BPS condition to the one found for five-dimensional static BPS black holes in 33 ,

$$
\epsilon_{i}=e^{\tilde{\sigma}} \epsilon_{(0) i},
$$

where $\epsilon_{(0) i}$ are constant spinors subject to the BPS condition $\gamma^{0} \epsilon_{(0) i}=\mp i \epsilon_{(0) i}$. This is of course the condition that we found earlier by looking for an eigenvector of the Bogomol'nyi matrix corresponding to massive pointlike BPS state. Moreover, for non-rotating, purely electric black holes, the condition imposed on the bosonic fields in five dimensions is 33

$$
\partial_{m} A_{0}^{I}= \pm \partial_{m}\left(e^{\tilde{\sigma}} h^{I}\right) .
$$

In terms of four-dimensional variables this becomes

$$
\partial_{m} b^{I}= \pm \partial_{m} \sigma^{I}
$$

which is indeed the same BPS condition as we found for rigid Euclidean vector multiplets.

Let us finally make a remark on the mass of the solitons and black holes obtained by lifting BPS instantons. For the general class of models the relation between soliton mass and instanton action was discussed previously. Moreover, it was shown in 26] that the instanton action and soliton mass are only finite if there is no contribution from the centers of the harmonic function. For metrics $N_{I J}=\partial_{I} \partial_{J} H(\sigma)$ with a homogenous Hesse potential, this requires that Hesse potential is either homogeneous of negative degree, or is the logarithm of a homogenous function of arbitrary degree. For rigid vector multiplets the Hesse potential and prepotential are related by [17, 26]

$$
F(X)=F(\sigma+e b)=4 H(\sigma+e b) .
$$

Since the prepotential, and, hence, the Hesse potential are homogeneous of degree three, we conclude that BPS solutions have infinite mass. Therefore these solutions are not instantons and solitons.

However for local vector multiplets, the four-dimensional prepotential $F(X)=$ $\left(X^{0}\right)^{-1} C_{I J K} X^{I} X^{J} X^{K}$ is related to the five-dimensional Hesse potential [17, 26]

$$
H(\sigma)=-\log C_{I J K} \sigma^{I} \sigma^{J} \sigma^{K}
$$


which is the logarithm of a homogeneous function. Thus in supergravity Euclidean BPS solutions have finite action and lift to BPS black holes of finite mass. Finally let us remark that for the Euclidean STU model, solutions can further be lifted to ten-dimensional five-branes [17. Therefore these solutions are relevant for understanding five-brane instantons in heterotic string theory.

\section{Discussion and Outlook}

In this paper we have discussed the relation between different Euclidean formulations of axions and their implications on instantons and solitons. We observed that the instanton action depends on the boundary values of the axionic fields, reflecting the breaking of continuous shift symmetries. This feature neither occurs in the dual tensor version of the theory nor in the dimensionally lifted theory. While there is no apparent contradiction in accepting that quantum theories can be 'equivalent up to zero modes', it becomes an issue as soon as one wants to compute physical effects due to instantons or solitons in a particular theory. Then one has to decide whether the physical theory one wants to study is a theory of axions or of antisymmetric tensor fields. This is relevant for the physics of wormholes, and also for the instanton calculus of string theory. According to $[8$, the formation of wormholes and baby universes only occurs naturally in axionic theories. Similarly, since the breaking of axionic shift symmetries is believed to be a non-perturbative effect relevant to low energy physics and hence phenomenology, the axionic formulation should also be fundamental for string effective theories. However, in practice the dual tensor field formulation is often used, and the programme of finding all non-perturbative corrections to hypermultiplets by using string dualities (reviewed in 32]) has so far relied on this. Further progress will require a better understanding of instantons within the axionic framework. We also remark that the vector multiplet instantons discussed in this paper correspond to five-brane instantons and world-sheet instantons of heterotic string compactifications [17. It would be interesting to use this for a direct check of heterotic-type II string duality.

One restriction that we imposed is that all shift symmetries commute. While this has the advantage that some conceptual aspects could be discussed very clearly, it is not the most general case of interest. The geometry of hypermultiplets provides an example of a more complicated class of geometries, with isometries forming a centrally extended Heisenberg group instead of an Abelian group. A generalized version of the c-map [35, in analogy to the generalized r-map introduced in 34, 26 should be the appropriate framework for investigating this larger class of models. One should then be able to tackle the issue raised in 28 systematically, namely which saddle points are really relevant for the semi-classical approximation. In the context of generating solitonic solutions from instantons, geometries of the type found in hypermultiplets allow to include magnetic in addition to electric charge.

While we have investigated the Euclidean formulation of axions, there are issues with the Euclidean formulation of fermions as well, in particular in the context of supersymmetric theories 21, 19, 22. We found that it is natural to complexify the axions, and in supersymmetric it then suggests itself to complexify all fields. For maximal eleven- and ten-dimensional supergravities the use of complexified fields leads to a simplified description of dualities, instantons and 
solitons 37 . In the appendix of 26 we gave an outline of how to complexify the scalar target spaces of the sigma models considered in this paper. For $N=2$ theories it should be useful to extend this to the whole theory, resulting in a 'complexified version' of special geometry. If one is interested in time-like Tduality transformations and type-II* string theories [38, the use of complexified fields should also be useful in Minkowski signature.

\section{References}

[1] P. Breitenlohner, D. Maison and G. Gibbons, Four-Dimensional Black Holes from Kaluza-Klein Theories, Commun. Math. Phys. 120 (1988) 295.

[2] K. Stelle, BPS branes in supergravity, Lecture Notes, hep-th/9803116.

[3] G. H. Derrick, Comments on nonlinear wave equations as models for elementary particles, J. Math. Phys. 5 (1964) 1252.

[4] S. Coleman, Aspects of Symmetry, Selected Erice lectures. Cambridge University Press, 1990.

[5] S. Giddings and A. Strominger, Axion-induced topology change in quantum gravity and string theory, Nucl. Phys. B 306 (1988) 890.

[6] D. Brown, C. Burgess, A. Kshirsagar, B. Whiting and J. York, Scalar Field Wormholes, Nucl. Phys. B 328 (1989) 213.

[7] S. Coleman and K. Lee, Wormholes made without massless matter fields, Nucl. Phys. B 329 (1990) 387.

[8] C. Burgess and A. Kshirsagar, Wormholes and duality, Nucl. Phys. B 324 (1989) 157.

[9] G. Gibbons, M. Green and M. Perry, Instantons and Seven-Branes in Type IIB Superstring Theory Phys. Lett. B 370 (1996) 37, hep-th/9511080.

[10] Effects of D-instantons, M. Green and M. Gutperle, Nucl. Phys. B 498 (1997) 195, hep-th/9701093.

[11] K. Behrndt, I. Gaida, D. Lüst, S. Mahapatra and T. Mohaupt, From type IIA black holes to T-dual type IIB D-instantons in $N=2, D=4$ supergravity Nucl. Phys. B 508 (1997) 659, hep-th/9706096.

[12] M. Gutperle and M. Spalinski, Supergravity instantons and the universal hypermultiplet JHEP 06 (2000) 037, hep-th/0005068.

[13] M. Gutperle and M. Spalinski, Supergravity instantons for $N=2$ hypermultiplets Nucl. Phys. B 598 (2001) 509, hep-th/0010192.

[14] U. Theis and S. Vandoren, Instantons in the double-tensor multiplet, JHEP 09 (2002) 059, hep-th/0208145.

[15] M. Davidse, M. de Vroome, U. Theis and S. Vandoren, Instanton solutions for the universal hypermultiplet, Fortsch. Phys. 52 (2004) 696, hep-th/0309220. 
[16] M. de Vroome and S. Vandoren, Supergravity description of spacetime instantons, Class. Quant. Grav. 24 (2007) 509, hep-th/0607055.

[17] T. Mohaupt and V. Cortés, Special Geometry of Euclidean Supersymmetry III, JHEP 07 (2009) 066, arXiv:0905.2844.

[18] B. Zumino, Euclidean supersymmetry and the many-instanton problem, Phys. Lett. B 69 (1977) 369.

[19] P. van Nieuwenhuizen and A. Waldron, On Euclidean spinors and Wick rotations, Phys. Lett. B 389 (1996) 29, hep-th/9608174.

[20] V. Cortés, C. Mayer, T. Mohaupt and F. Saueressig, Special Geometry of Euclidean Supersymmetry I, JHEP 03 (2004) 028, hep-th/0312001.

[21] H. Nicolai, A Possible constructive approach to Super $\phi^{3}$ in four- dimensions. 1. Euclidean formulation of the model, Nucl. Phys. B 140 (1978) 294.

[22] P. van Nieuwenhuizen and A. Waldron, A continuous Wick rotation for spinor fields and supersymmetry in Euclidean space, hep-th/9611043.

[23] M. Blau and G. Thompson, Euclidean SYM theories by time reduction and special holonomy manifolds, Phys. Lett. B 415 (1997) 242, hep-th/9706225.

[24] A. Belitsky, S. Vandoren and P. van Nieuwenhuizen, Instantons, Euclidean supersymmetry and Wick rotations, Phys. Lett. B 477 (2000) 335, hep-th/0001010.

[25] U. Theis and P. van Nieuwenhuizen, Ward identities for $N=2$ rigid and local supersymmetry in Euclidean space, Class. Quant. Grav. 18 (2001) 5469, hep-th/0108204.

[26] T. Mohaupt and K. Waite, Instantons, black holes and harmonic functions, JHEP 10 (2009) 058, arXiv:0906.3451.

[27] T. Mohaupt and O. Vaughan, Non-extremal Black Holes, Harmonic Functions, and Attractor Equations, Class. Quant. Grav. 27 (2010) 235008, arXiv:1006.3439

[28] M. Chiodaroli and M. Gutperle, Instantons and Wormholes in N=2 supergravity Phys. Rev D 79 (2009) 085023, arXiv:0901.1616.

[29] B. Eynard, Large $N$ expansion of convergent matrix integrals, holomorphic anomalies, and background independence, arXiv:0802.1788

[30] C.M. Hull, A geometry for non-geometric string backgrounds, JHEP 10 (2005) 065, hep-th/0406102.

[31] R. Siebelink, The low-energy effective action for perturbative heterotic strings on $K(3) \times T^{2}$ and the $d=4 N=2$ vector-tensor multiplet, Nucl. Phys. B 512 (1998) 148, hep-th/9709129.

[32] F. Saueressig, Recent results in four-dimensional non-perturbative string theory, J. Phys. Conf. Ser. 110 (2008) 102010, arXiv:0710.4931. 
[33] A.H. Chamseddine and W. Sabra, Metrics admitting Killing spinors in five dimensions, Phys. Lett. B 426 (1998) 36, hep-th/9801161.

[34] A. Alekseevski and V. Cortés, Geometric construction of the r-map: from affine special real to special Kähler manifolds, arXiv:0811.1658.

[35] S. Ferrara and S. Sabharwal, Quaternionic Manifolds for Type II Superstring Vacua of Calabi-Yau Spaces, Nucl. Phys. B 332 (1990) 317.

[36] M. Gunaydin, G. Sierra and P.K. Townsend, The Geometry of N=2 Maxwell-Einstein Supergravity and Jordan Algebras Nucl. Phys. B 242 (1984) 244.

[37] E. Bergshoeff, J. Hartong, A. Ploegh, J. Rosseel and D. Van den Bleeken, Pseudo-supersymmetry and a tale of alternate realities, JHEP 07 (2007) 067, arXiv:0704.3559

[38] C.M. Hull, Timelike T-duality, de Sitter space, large $N$ gauge theories and topological field theory JHEP 07 (1998) 021, hep-th/9806146.

[39] P.K. Townsend, M-theory from its superalgebra, Lecture Notes, hep-th/9712004.

[40] M. Gunaydin, A. Neitzke, B. Pioline and A. Waldron, Quantum Attractor Flows, JHEP 09 (2007) 056, arXiv:0707.0267.

[41] T. Mohaupt, Instanton solutions for Euclidean $N=2$ vector multiplets, Fortsch. Phys. 56 (2008) 480.

[42] M. Henneaux and C. Teitelboim, Quantization of Gauge Systems, Princeton University Press, 1992.

[43] N. Mohammedi, R.T. Moss and R.D. Simmons, A Dual description of the four-dimensional nonlinear sigma model, Z. Phys. C 75 (1997) 173.

[44] A. Ceresole and G. Dall'Agata, Flow Equations for Non-BPS Extremal Black Holes, JHEP 03 (2007) 110, hep-th/0702088.

[45] G. Lopes Cardoso, A. Ceresole, G. Dall'Agata, J.M. Oberreuter and J. Perz, First-order flow equations for extremal black holes in very special geometry, JHEP 10 (2007) 063, arXiv:0706.3373. 